\title{
Gas Turbulence Modulation in a Two-fluid Model for Gas-Solid Flows
}

\author{
Yonghao Zhang a and Jason M Reese ${ }^{\mathrm{b}}$ \\ a Computational Science and Engineering Department, CLRC Daresbury Laboratory, \\ Warrington, WA4 4AD, UK \\ b Department of Mechanical Engineering, University of Strathclyde, 75 Montrose Street, \\ Glasgow G1 1XJ, UK
}

\begin{abstract}
Recent rapid progress in the theoretical and experimental study of turbulence modulation has led to greater understanding of the physics of particle-gas turbulence interactions. In this paper we establish a new two-fluid model incorporating these advances for relatively dilute gas-solid flows containing high-inertia particles. The effect of aerodynamic forces upon the particulate stresses is considered in this kinetic theory-based model, and the influence of the particles on the turbulent gas is addressed: the work associated with drag forces contributes to the gas turbulent energy, and the space occupied by particles restricts the turbulent length scale. The inter-particle length scale, which is usually ignored, has been incorporated into a new model for determining the turbulent length scale. This model also considers the transport effect on the turbulent length scale. Our simulation results for fully-developed steady flows in vertical pipes are compared with a wide range of published experimental data and, generally, good agreement is shown. This comprehensive and validated model accounts for many of the inter-phase interactions that have been shown to be important.
\end{abstract}

keywords: gas-solid flows, granular flows, two-fluid model, kinetic theory, turbulence modulation, particulate flows 


\section{Introduction}

Depending on the solid volume fraction, flows of solid particles in a gas can be classified into three regimes: "one-way", "two-way" and "four-way" coupling (Elghobashi, 1994). When the solid volume fraction is less than about $10^{-6}$, the particles have negligible effect on the gas turbulence, which is termed one-way coupling. When the solid volume fraction is larger than about $10^{-6}$, the particles will either enhance or attenuate the gas turbulence; this is two-way coupling. Once the solid volume fraction increases beyond $0.1 \%$, collisions also begin to play an important role in the flows, which is called four-way coupling. In a very dense flow the carrier gas turbulence may be ignored. However, in the regime of solid volume fraction greater than $10^{-6}$, but not high enough that the gas turbulence can be neglected, we may have to consider not only the collisions between particles but also the turbulent interactions between the two phases. This paper examines these issues in this flow regime.

\section{Turbulence modulation}

The modulation of the gas turbulence in particle-laden flows is not well understood. However, significant advances, through both experimental measurements and direct numerical simulation (DNS), have been achieved towards an appreciation of the mechanisms behind this modulation. Many factors, such as particle size, density and volume fraction have been identified as relevant. Generally, experimental data show that larger particles (i.e. when the ratio of particle diameter to pure gas turbulent length scale, $d / l_{i}$, is greater than about $0.1)$ tend to enhance the turbulence and smaller particles $\left(d / l_{i} \leq 0.1\right)$ attenuate it (Gore and Crowe, 1989). This turbulence modulation may be due to, amongst other mechanisms, the wakes of particles, the displacement of the flow field by particles, turbulent energy transfer, and modification of velocity gradients (Crowe, 1997). Therefore, it is timely to begin exploring new turbulence modulation models based on these phenomena. 
Yuan and Michaelides (1992) argued that the wake is responsible for the augmentation of turbulence and the work done on the particles is responsible for the attenuation of turbulence. This approach was developed and extended by Yarin and Hetsroni (1994), who proposed a more detailed expression for the wake. Bolio and Sinclair (1995) adopted the original model of Yuan and Michaelides, and confirmed that the wake enhanced gas turbulence. However, it was experimentally observed that turbulent energy may also increase when the particle Reynolds number is small and the wakes are negligible (Hardalupas et al., 1989). Kenning and Crowe (1997) suggested that the work done by the particles on the gas via drag could generate fluid turbulence. Crowe and Gillandt (1998), Crowe and Wang (2000) and Crowe (2000) derived and improved a detailed turbulence modulation model following the work of Kenning and Crowe (1997). These authors argued that the common approach to the derivation of the turbulent kinetic energy balance equation (e.g. Louge et al., 1991), which treats the averaged velocity as if it were a local velocity in the momentum equations of both phases, is not appropriate. In other words, the turbulent kinetic energy equation should be derived from the instantaneous Navier-Stokes equation, which does not include a coupling drag force term. Fessler and Eaton (1999) also pointed out that previous models which used an extra turbulent energy source or sink to represent the influence of the particles did not fully capture the physics of particle-gas interactions.

Rigorously deriving a turbulence model, even for a single-phase flow, is extremely problematic, so all gas turbulence modulation models inevitably require some ad hoc assumptions. We can, however, make some general remarks when considering current models. As the $k-\varepsilon$ model has been successfully and widely used for single-phase flows, it has consequently found many advocates in the gas-solid flow community (see, e.g., Mostafa and Mongia, 1988; Rizk and Elghobashi, 1989; Thai Van et al., 1994; Bolio and Sinclair, 1995; Bolio et al., 1995; Cao and Ahmadi, 1995; Dasgupta et al., 1994, 1998; Neri and Gidaspow, 
2001). However, the $k$-equation in these models is physically insufficient, as pointed out by Crowe and coworkers. In our previous research (Zhang and Reese, 2001), the $k$-equation of Crowe et al. was numerically tested; however in this model a prescribed turbulence length scale is used. Recently, the importance of the inter-particle length scale on the gas turbulence has also been experimentally confirmed (Sato et al., 2000), which has always previously been neglected in the turbulence modeling. A more rigorous transport equation for the turbulent length scale is needed to address the influence of the particles on the turbulent length scale.

Drawing on experimental results for sediment-laden channel flows, Kovacs (1998) argued that the mixing length is restricted by the space occupied by the suspended particles, which is not available to the gas phase. From the original concept of the mixing length of the gas turbulence, we interpret the presence of particles as inhibiting the free random motion of the gas molecular clusters, which leads to a reduction of the turbulent length scale. In this paper, we therefore construct a new model which not only accounts for the transport effect but also the inter-particle spacing on the turbulent length scale.

Most previous work simply adopted exactly the same values for the necessary turbulence model constants as in single-phase turbulent flow. However, Squires and Eaton (1994) have demonstrated that the values of these constants are at least related to the ratio of mean particle relaxation time, $t_{12}^{x}$, to the time scale of large eddies, $t_{1}^{t}$, and the solid volume fraction (using the notation of Peirano and Leckner, 1998). Based on the direct simulation results of Squires and Eaton (1994), we address the effect of the solid volume fraction of high-inertia particles (i.e. when $t_{12}^{x} \gg t_{1}^{t}$ ) on these essential two-equation turbulent model constants.

\section{Particulate stresses}

In a continuum model for a dispersed phase, the particulate stresses are formulated by 
appropriate constitutive laws. These stresses are generated by direct particle-particle interactions and particle random motion. Currently, there are two approaches: traditional empirical models, and a kinetic theory of granular flow. The former models are relatively straightforward to apply in numerical simulations, but have certain arbitrary adjustable parameters. The kinetic theory of granular flow is more rigorous, but can be criticised as unsuitable because of the physical difference between a dry (no interstitial gas) granular system and a gas-solid two-phase system (Buyevich, 1999). In a gas-solid flow, the particles are generally driven by the gas phase via the inter-phase forces. The influence of the gas phase on the constitutive laws for the particulate phase is ignored in a kinetic theory of dry granular flow, but if the carrier gas can be incorporated this would be a powerful tool for obtaining suitable constitutive equations for the dispersed phase.

Following the pioneering work of Bagnold (1954), Savage and Jeffrey (1981) drew comparison between the random motion of particles and that of gas molecules, and borrowed ideas from the kinetic theory of gases to model granular flow. Since then, many competing theories have been proposed (e.g. Ogawa et al., 1980; Jenkins and Savage, 1983; Lun et al., 1984; Jenkins and Richman, 1985; Abu-Zaid and Ahmadi, 1990; Gidaspow, 1994). Sinclair and Jackson (1989) used the kinetic theory of dry granular flow to model the particulate stresses in a gas-solid system, and this has been widely adopted (e.g. Bolio and Sinclair, 1995; Cao and Ahmadi, 1995; Dasgupta et al., 1994, 1998; Neri and Gidaspow, 2001). However, the kinetic theory of dry granular flows may not be appropriate for the particulate phase in a relatively dilute gas-solid system.

The interstitial fluid is often discounted in a kinetic theory model by assuming that the random motion of particles is controlled by the inter-particle collisions rather than the interstitial fluid flow. This assumption is acceptable if the mean particle relaxation time, $t_{12}^{x}$, is much larger than the mean particle collision time, $t_{2}^{c}$, and also larger than the time scale of 
large eddies, $t_{1}^{t}$. However, in a relatively dilute flow with small particles, $t_{12}^{x}$ is of the same order as $t_{1}^{t}$ or $t_{2}^{c}$ (or the time scale associated with particle-wall collisions if it is smaller), and the random free motion of the particles will be affected by both the gas turbulent fluctuations and its mean flow. In this case, the influence of the gas flow should be incorporated in the constitutive formulations for the particulate stresses. Therefore, previous models based on a kinetic theory of dry granular flows are not suitable for the relatively dilute flows we examine in this paper.

The effect of the interstitial fluid has been studied by many researchers (e.g. Ma and Ahmadi, 1988; Koch, 1990; Gidaspow, 1994; Balzer et al., 1995; Boelle et al., 1995; Koch and Sangani, 1999; Agrawal et al., 2001). Zaichik et al. (1997) proposed a generalized kinetic model which accounted for both inter-particle collisions and particle-turbulence interactions by using a Boltzmann integral operator and a generalized Fokker-Planck differential operator respectively. Peirano and Leckner (1998) derived a competing kinetic theory incorporating interstitial gas, based on the work of Jenkins and Richman (1985). If the interstitial gas were omitted, their results are identical to the work of Jenkins and Richman (1985) and Lun et al. (1984). Although their model is restricted to only slightly inelastic particle-particle collisions, it does account for the effect of both the gas fluctuational motion and mean flow on the particles. In this paper, we will use the model of Peirano and Leckner for the particulate phase to address and numerically assess the gas turbulence effect on the constitutive laws for the particulate stresses. As far as the authors are aware this is the first thorough numerical examination of the theory proposed by Peirano and Leckner.

Inelastic collisions between particles cause spatial inhomogeneities in the particulate phase flowfield, so, properly, the mesoscopic nature of the gas-solid system should be considered. However, here we focus on dilute flows in pipes which are of small enough diameter that the spatial inhomogeneity of the particulate phase flow structure can be 
regarded as small. We therefore assume the fully-developed turbulent gas-solid flows are steady and axi-symmetric. The limits of validity of this approach can be ascertained by numerical simulation and comparison with a range of experimental data.

\section{Outline of this paper}

We begin with the governing equations for a two-fluid model and then introduce the essential components of our model one by one. The inter-phase momentum correlation, i.e. the drag force, is modeled by considering the effect of solid particle fluctuations. The gas turbulence modulation due to the particles is addressed, and a new two-equation turbulence model is introduced which accounts for the effect of inter-particle spacing. The influence of particles on the $k$ - $\varepsilon$ turbulence model parameters is tackled and the constitutive equations for the particulate stresses, which account for the effect of the gas turbulence, are given. Finally, the two main competing boundary conditions of the particulate phase are discussed. Our numerical simulation results for relatively dilute gas-solid flows in vertical pipes are then discussed and compared with experimental data.

\section{The mathematical model}

In a two-fluid model, the governing equations for a dispersed solid phase and a carrier gas phase are locally-averaged, and both expressions have the same general form. The momentum equations can be given as (Anderson and Jackson, 1967; Jackson, 1997):

solid phase

$$
\varepsilon_{2} \rho_{2} \frac{D}{D t} \boldsymbol{U}=-\varepsilon_{2} \nabla P-\nabla \cdot \tau_{2}+\boldsymbol{F}_{d r a g}+\varepsilon_{2} \rho_{2} \boldsymbol{g}
$$

gas phase

$$
\varepsilon_{1} \rho_{1} \frac{D}{D t} \boldsymbol{V}=-\varepsilon_{1} \nabla P-\nabla \cdot \tau_{1}-\boldsymbol{F}_{d r a g},
$$


where subscripts 1 and 2 represent the gas phase and solid phase respectively, i.e. $\varepsilon_{1}$ and $\varepsilon_{2}$ are the volume fractions of the gas phase and the particulate phase; $\rho$ is the density; $\tau$ is the stress; $\boldsymbol{U}$ is the averaged velocity of the solid phase; $\boldsymbol{V}$ is the averaged velocity of the gas phase; $\boldsymbol{F}_{d r a g}$ is the averaged drag force; $\boldsymbol{g}$ is the gravitational acceleration. The essence of a two-fluid model is accurately to model particulate and gas stresses, $\tau_{1}$ and $\tau_{2}$, and drag force, $\boldsymbol{F}_{\text {drag }}$.

\section{The drag force}

For a particle moving in a cloud of particles, the particle volume fraction has been shown to have an important effect on the drag force (e.g. Wen and Yu 1966; Di Felice 1994). The drag force acting on a single particle can be given as $\boldsymbol{f}_{\text {drag }}=\beta(\overline{\boldsymbol{v}}-\boldsymbol{u})$, where $\overline{\boldsymbol{v}}$ is the volumeaveraged gas velocity on the control volume containing only a single particle, and $\boldsymbol{u}$ is the instantaneous velocity of the particle. A correction factor $f\left(\varepsilon_{2}\right)$ is usually adopted to address the effect of particle volume fraction on the momentum transfer coefficient, i.e. $\beta=\frac{3}{4} C_{D} \frac{\varepsilon_{2} \rho_{1}}{d}|\overline{\boldsymbol{v}}-\boldsymbol{u}| f\left(\varepsilon_{2}\right)$. Many forms for $f\left(\varepsilon_{2}\right)$ have been proposed, for example Wen and Yu (1966) gave $f\left(\varepsilon_{2}\right)=\left(1-\varepsilon_{2}\right)^{-2.65}$.

However, the two-fluid model as derived by Anderson and Jackson (1967) is based on a control volume which contains statistically many particles, and the drag force is averaged over a number of particles rather than a single particle control volume. Although the voidage effect has usually been addressed, the effect of the random motion of the particles has not been sufficiently implemented in the model. For example, the widely used phenomenological Ergun formula is originally based on observations on a fixed bed where the voidage is the main concern. For freely and randomly moving particles in a two-fluid model, not only the 
particle volume fraction but also the random fluctuational motion of individual particles needs to be considered.

By accounting for the effect of the random motion of particles, we have derived an expression for the averaged drag force (Zhang and Reese, 2003):

$$
\boldsymbol{F}_{\text {drag }}=\beta_{0}(\boldsymbol{V}-\boldsymbol{U})
$$

where,

$$
\beta_{0}=\frac{3}{4} C_{D} \frac{\varepsilon_{2} \rho_{1}}{d} \varepsilon_{1}^{-2.65} U_{r}, \quad \varepsilon_{1} \geq 0.8
$$

and $\beta_{0}$ is the effective inter-phase momentum transfer coefficient for drag. The mean slip velocity, $U_{r}$, is

$$
U_{r}=\sqrt{(\boldsymbol{V}-\boldsymbol{U})^{2}+\frac{8 T}{\pi}},
$$

which accounts for both the slip of mean velocities between the two phases and the relative velocity between the random motion of the particles and the mean flow of the gas. The granular temperature $T$ is defined as $\left\langle\boldsymbol{u}^{\prime} \cdot \boldsymbol{u}^{\prime}\right\rangle / 3$ where $\boldsymbol{u}^{\prime}$ is the fluctuational velocity of a particle. When using correlations to obtain the drag coefficient in equation (4), an averaged particle Reynolds number should be employed, viz.

$$
\operatorname{Re}_{p}=\frac{\rho_{1} U_{r} d}{\mu}
$$

In our formulation, the mean relative fluctuational velocity of particles in a control volume is accounted for, which is close to $\sqrt{8 T / \pi}$. Although any change in the form of the correction factor $f\left(\varepsilon_{2}\right)$ may well offset the relatively small effect of the random motion of particles on the momentum transfer coefficient, this fundamental mechanism still represents a physical effect which should be incorporated within any physically-consistent two-fluid 
modeling methodology. This model has been shown to have an appreciable effect on simulation results for benchmark cases (Zhang and Reese 2003).

\section{Gas turbulence modulation}

In the widespread $k$ - $\varepsilon$ eddy viscosity turbulence model for a gas phase, the gas effective stress consists of a viscosity stress and a Reynolds turbulent stress, i.e.

$$
\tau_{1 i j}=\left(\mu+\mu_{t}\right)\left(\frac{\partial v_{i}}{\partial x_{j}}+\frac{\partial v_{j}}{\partial x_{i}}\right)-\frac{2}{3}\left(\mu+\mu_{t}\right) \nabla \cdot v \delta_{i j}-\frac{2}{3} \rho_{1} k \delta_{i j}
$$

where $\mu_{t}$ is the eddy viscosity; the turbulent kinetic energy, $k$, is $1 / 2\left\langle v^{\prime} \cdot v^{\prime}\right\rangle$, and $v^{\prime}$ is the fluctuational velocity of the gas. The expression for $k$ for a single fluid flow can be derived from the Navier-Stokes equations. In a similar way to a single-phase flow, the turbulent energy equation for the gas in a two-phase system can be given as:

$$
\frac{\partial}{\partial t}\left(\varepsilon_{1} \rho_{1} k\right)+\frac{\partial}{\partial x_{j}}\left(\varepsilon_{1} \rho_{1} V_{j} k\right)=\frac{\partial}{\partial x_{j}}\left[\varepsilon_{1}\left(\mu+\frac{\mu_{t}}{\sigma_{k}}\right) \frac{\partial k}{\partial x_{j}}\right]-\varepsilon_{1} \rho_{1}\left\langle v_{i}^{\prime} v_{j}^{\prime}\right\rangle \frac{\partial}{\partial x_{j}} V_{i}+\Delta k-\varepsilon_{1} \rho_{1} \varepsilon
$$

where $\Delta k$ is a source or sink of turbulent kinetic energy due to the presence of particles, $\varepsilon$ is the dissipation rate of the kinetic energy and $\sigma_{k}$ is a Prandtl number relating the diffusion of $k$ to the eddy viscosity.

The commonly-used expression for $\Delta k$ is (e.g. Chen and Wood, 1985; Berlemont et al., 1990; Louge et al., 1991)

$$
\Delta k=\beta_{0}\left(\left\langle\boldsymbol{u}^{\prime} \cdot \boldsymbol{v}^{\prime}\right\rangle-2 k\right)
$$

However, simulations using this model of $\Delta k$ have been found to underestimate the gas kinetic energy somewhat (Louge et al., 1991; Bolio et al., 1995). Other turbulent energy generation mechanisms, such as particle wakes, may need to be incorporated (e.g. Yuan and Michaelides, 1992; Yarin and Hetsroni, 1994; Bolio and Sinclair, 1995; Sinclair and Mallo, 1998). Crowe and Gillandt (1998) argued that, in any case, the coupling term is not correctly 
modeled in equation (9) because the coupling drag force is an averaged value which cannot be regarded as a point-value in the derivation of equation (8). Their model asserted, instead,

$$
\Delta k=\beta_{0}|U-V|^{2}+\beta_{0}\left(3 T-k_{12}\right),
$$

where $k_{12}=\left\langle\boldsymbol{u}^{\prime} \cdot \boldsymbol{v}^{\prime}\right\rangle$, an estimate for which is given in Zhang and Reese (2001):

$$
k_{12} \approx \frac{\beta_{0} U_{r}^{2} t_{2}^{c}}{\rho_{2} v_{2}} .
$$

Here, $k_{12}$ relates to the auto-correlation of the force experienced by a representative particle. If the correlation time is taken to be comparable to the collision interval and the force acting on a particle is the drag force, then it is obtained by an averaging process.

The disagreement between equations (9) and (10) stems from the different averaging processes: a volume and temporal double average is used in deriving equation (9) and only volume averaging is used in the model of Crowe and Gillandt. Both equations have been numerically evaluated in Zhang and Reese (2001), and the model of Crowe and Gillandt has shown better overall performance in terms of accuracy. Therefore, we will adopt equation (10) with equation (11) in the present paper.

Although Crowe and Gillandt have rigorously derived a $k$-equation for gas solid flows, they used a prescribed hybrid turbulent dissipation length scale to account for the presence of particles, i.e.

$$
l_{h}=\frac{l \lambda}{l+\lambda}
$$

where $\lambda$ is the inter-particle spacing. This approach regards the inter-particle space as an important equivalent characteristic turbulence length scale, restricting the turbulent fluctuations. A similar idea can also be found in the work of Kovacs (1998). However, the transport effects on the turbulent length scale are as important as those on the turbulent kinetic energy (Launder and Spalding, 1972). Although the variation of the turbulent length 
scale, $l$, needs to be determined by a transport equation, $l$ itself is not necessarily a dependent variable.

Here we propose a new transport equation to solve for the turbulent energy dissipation rate, $\varepsilon$. The turbulent length scale, $l$, can therefore be determined via a new relation to the turbulent energy dissipation rate, $\varepsilon$, to account for the effect of the inter-particle length scale. In a similar way to that for a single phase flow, the new $\varepsilon$-equation is constructed as:

$$
\begin{aligned}
\frac{\partial}{\partial t}\left(\varepsilon_{1} \rho_{1} \varepsilon\right)+\frac{\partial}{\partial x_{j}}\left(\varepsilon_{1} \rho_{1} V_{j} \varepsilon\right)= & \frac{\partial}{\partial x_{j}}\left\{\varepsilon_{1}\left(\mu+\frac{\mu_{t}}{\sigma_{\varepsilon}}\right) \frac{\partial \varepsilon}{\partial x_{j}}\right\}+c_{1} \varepsilon_{1} \frac{\varepsilon}{k} \mu_{t}\left(\frac{\partial V_{i}}{\partial x_{j}}+\frac{\partial V_{j}}{\partial x_{i}}\right) \frac{\partial V_{i}}{\partial x_{j}} \\
& -c_{2} \rho_{1} \varepsilon_{1} \frac{\varepsilon^{2}}{k}+c_{3}\left[\beta_{0}|U-V|^{2}+\beta_{0}\left(3 T-k_{12}\right)\right] \frac{\varepsilon}{k} .
\end{aligned}
$$

where $\sigma_{\varepsilon}$ is the Prandtl number relating the diffusion of $\varepsilon$ to the eddy viscosity, which may be given as:

$$
\mu_{t}=C_{\mu} \rho_{1} k^{2} / \varepsilon
$$

where $C_{\mu}$ is a constant parameter. In previous turbulent modulation models, the turbulent length scale, $l$, is usually the same as in a gas single-phase turbulent flow (e.g. Mostafa and Mongia, 1981), i.e. through

$$
\varepsilon=C_{\mu}^{3 / 4} k^{3 / 2} / l
$$

However, the importance of inter-particle space to the turbulent length scale has been neglected in this equation. Now, we need to incorporate this effect into a new model for the turbulent length scale.

In a mixing length turbulence model, the turbulent length scale is analogous to the free path of the random motion of gas molecules. The presence of particles "blocks" the free path of gas molecular clusters, leading to a reduced turbulent length scale (Kovacs, 1998). Suppose there are $n$ particles each of diameter $d$ in the local element volume of $l_{s}^{3}\left(l_{s}\right.$ is the 
length scale of this element volume), where $n$ is statistically sufficiently large. The space occupied by particles is not available to the gas. This solid-occupied space is given by

$$
\frac{\pi}{6} d^{3} n=\varepsilon_{2} l_{s}^{3}
$$

If the particles are distributed evenly in the $x, y$ and $z$ directions, then the effective length scale can be reduced to:

$$
l_{e}=\left(1-n^{\frac{1}{3}} d\right)=\left\{1-\left(\frac{6 \varepsilon_{2}}{\pi}\right)^{\frac{1}{3}}\right\} l_{s} .
$$

This can be better illustrated by an example. Suppose there are 1000 particles in the element volume, distributed evenly $10 \times 10 \times 10$ in the $x, y, z$ directions. In each direction, the dimensional space occupied by the particles is $10 d$ in this case (generally, $n^{1 / 3} d$ ). Deducting this length scale occupied by the particles from the total available space, we obtain equation (17).

Because the turbulence of the gas will be effectively suppressed at the close-packed limit, then the reduction of the turbulence length scale should be normalized by the highest solid volume fraction limit in which turbulence disappears. This is common practice in this field (see, e.g. Cao and Ahmadi, 1995): an analogous example is the expression for the radial distribution function, which is also widely taken to involve the close packed solid volume fraction even for very dilute flows (e.g. Neri and Gidaspow, 2001). Because the space occupied by the spherical particles is not available to the gas molecular clusters, and the gas turbulence is likely to be negligible at solid volume fractions even somewhat below the closely-packed limit, the reduced turbulent length scale, $l_{r}$, becomes

$$
l_{r}=\left[1-\left(\frac{6 \varepsilon_{2}}{\pi \varepsilon_{2 m}}\right)^{1 / 3}\right] l,
$$


where $\varepsilon_{2 m}$ represents the random close-packing particle volume fraction, which is 0.64 . We put forward equation (18) as a suitable physical model in the dilute flow regime examined here.

Therefore, the new relation between turbulent energy dissipation rate and turbulent length scale becomes,

$$
\varepsilon=\frac{C_{\mu}^{3 / 4}}{1-\left(6 \varepsilon_{2} / \pi \varepsilon_{2 m}\right)^{1 / 3}} \frac{k^{3 / 2}}{l} .
$$

As this equation fails when $\varepsilon_{2}$ exceeds 0.34 , a consequent assumption is that the gas turbulence can be ignored at this high solid volume fraction. Equation (19) accounts for the effect of particles on the turbulent length scale. In the relatively dilute limit we examine, the normalizing effect of the maximum volume fraction is significant. Our model's sensitivity to this parameter will be examined in section 5 below, where the significance of this turbulence modulation, even at such small solid volume fractions, will be shown. Anticipating this, we contend that the effect of solid volume fraction should be included in a comprehensive and mechanistic gas-solid flow model.

\section{The turbulence model parameters}

The last term in equation (13) is an ad hoc parameterization of the effect of particles on $\varepsilon$ (Squires and Eaton, 1994). All the model constants except $c_{3}$ are normally adopted straight from a single-phase fluid turbulence model. Drawing on the comparative study of Hrenya et al. (1995) for single-phase fully-developed steady flows in vertical pipes, the most appropriate constants are those proposed by Rodi and Mansour (1993) and Michelassi et al. (1993), viz. $C_{\mu}=0.09 ; c_{1}=1.44 ; c_{2}=1.92 ; \sigma_{k}=1.3 ; \sigma_{\varepsilon}=1.3$, which give good agreement with single-phase experimental measurements, as shown in figure 1 . The constant $c_{3}$ is a tunable parameter which is usually optimized to provide agreement with a specific experiment, 
varying between 1.0 and 2.0. This may indicate that it could depend on other factors such as particle size, density and volume fraction. As Squires and Eaton (1994) reported, generally $c_{2}$ and $c_{3}$ both depend on mass loading and the ratio of particle relaxation time to large eddy time scale.

In a $k-\varepsilon$ model, the characteristic time scale of the large eddies, $t_{1}^{t}$, can be given by $C_{\mu} k / \varepsilon$; the particle relaxation time, $t_{12}^{x}$, by $4 d \rho_{2} / 3 \rho_{1} C_{D} U_{r}$; and the particle collision time, $t_{2}^{c}$, by $d / 24 \varepsilon_{2} \chi \sqrt{\pi / T}$. The radial distribution function, $\chi$, is described here by the expression of Lun and Savage (1987), viz. $\chi=\left[1-\varepsilon_{2} / \varepsilon_{2 m}\right]^{-2.5 \varepsilon_{2 m}}$. For light particles $t_{12}^{x} / t_{1}^{t}$ is $\mathrm{O}(1)$ or less, and mass loading has a strong effect on the value of $c_{2}$ and $c_{3}$. But for the heavier particles we consider, $t_{12}^{x} / t_{1}^{t}$ is $\mathrm{O}(10)$ or more and the influence of mass loading on $c_{2}$ may be negligible, while $c_{3}$ decreases with increasing mass loading. Therefore, the value of $c_{2}$ will be taken to be the same as in pure gas turbulent flow, but we propose a heuristic variable $c_{\varepsilon 3}$, which is a function of solid volume fraction:

$$
c_{\varepsilon 3}=\left[1-\left(\frac{6 \varepsilon_{2}}{\pi \varepsilon_{2 m}}\right)^{\frac{1}{3}}\right] c_{3},
$$

to replace $c_{3}$ in the term in equation (13) for the particle-generated turbulent source or sink. While the flow is dilute, the value of $c_{\varepsilon 3}$ will decrease as the solid volume fraction increases towards the random close-packed limit. Because any turbulence model is based on hypotheses of turbulent motion and requires empirical input, $c_{3}$ is such an empirical constant. Our modification to $c_{3}$, as given by equation (20), is based on observations from the DNS database of Squires and Eaton (1994) and requires testing.

At the moment, there are no universal turbulence models available with clearly defined values of the parameters. The effect of particles on $c_{3}$ is always neglected in the literature, and its tuned value varies widely from 1.0 to 2.0 . The very common and sensible 
approach is to tune this value for a benchmark case, and then fix and use this value to examine other cases to see whether quantitative agreement can still be achieved in these different cases. Here, the value of $c_{3}=1.95$ in equation (20) is obtained by benchmarking against a single data set of Tusji et al. (1984). This value is fixed to test Tsuji's other data sets, and also the data sets from different experiments (Maeda et al., 1980; Lee and Durst, 1982). The solid volume fraction dependent $c_{\varepsilon 3}$ is then found to vary between 1.4 to 1.7 within calculations.

In each case our new model using $c_{\varepsilon 3}$ shows good agreement without the need for further tuning to agree with each individual case. This may indicate our empirical modification of $c_{3}$ does reflect the particle effect. The sensitivity of our model to the value of $c_{3}$ is shown below in figures 24 and 25 , where significantly different predictions for $k$ are found for different values of $c_{3}$ in equation (20). By incorporating the effect of particle volume fraction, as given by equation (20), we achieve substantially more accurate and (as we only tune a single parameter once) more robust and practical simulation results overall.

\section{The particulate stresses}

The solid stress has been confirmed to be important even in a dilute flow (e.g. Pita and Sundaresan, 1991; Dasgupta et al., 1994, 1998). Although the kinetic theory of dry granular flow is widely used to describe the solid stress, this has been criticized as being ill-founded because, as inter-particle collisions are inelastic, the only state of equilibrium for unforced granular flows is that of zero granular temperature (Goldhirsch and Zanetti, 1993; Kadanoff, 1999). However, in a gas-solid dilute flow, the equilibrium state of the granular solid phase depends on the trade-off between inelastic dissipation and particle-gas interactions. The interstitial gas will affect the random motion of particles, so that solid phase fluctuations will be correlated to the gas fluctuations and influenced by the mean gas flow. Moreover, the 
inelastic kinetic energy dissipation is small in a relatively dilute flow. As a result, the kinetic theory approach could capture a main generating mechanism of the solid stress in a gas-solid system, provided the interstitial gas can be properly considered.

Peirano and Leckner (1998) addressed the turbulent gas effect on the solid stress. Here, as in Zhang and Reese (2001) previously, we will adopt their work to model the solid stress. For the sake of completeness, we briefly summarise this model as:

$$
\tau_{2 i j}=\left(P_{2}-\zeta_{2} S_{k k}\right) \delta_{i j}-2 \varepsilon_{2} \rho_{2}\left(\psi_{2}^{c}+\psi_{2}^{t}\right) \hat{S}_{i j}
$$

where the particle normal stress is the same as in the kinetic theory model of granular flow, i.e.

$$
P_{2}=\varepsilon_{2} \rho_{2}\left(1+2 \varepsilon_{2} \chi(1+e)\right) T
$$

where $S_{2 i j}=\left(U_{i, j}+U_{j, i}\right) / 2, \hat{S}_{2 i j}=S_{2 i j}-S_{2 m m} \delta_{i j} / 3$ and $e$ is the particle-particle collisional coefficient of restitution; $\zeta_{2}, \psi_{2}^{c}$ and $\psi_{2}^{t}$ are the bulk viscosity, the collisional viscosity and the turbulent viscosity, respectively, given by

$$
\begin{aligned}
& \zeta_{2}=\frac{4}{3} d \varepsilon_{2}^{2} \rho_{2} \chi(1+e) \sqrt{\frac{T}{\pi}}, \\
& \psi_{2}^{c}=\frac{4}{5} \varepsilon_{2} \chi(1+e)\left(\psi_{2}^{t}+d \sqrt{\frac{T}{\pi}}\right), \\
& \psi_{2}^{t}=\left(\frac{2}{3} \frac{t_{12}^{t}}{t_{12}^{x}} k_{12}+\left(1+\varepsilon_{2} \chi A\right) T\right) /\left(\frac{2}{t_{12}^{x}}+\frac{B}{t_{2}^{c}}\right),
\end{aligned}
$$

where $A=\frac{2}{5}(1+e)(3 e-1)$ and $B=\frac{1}{5}(1+e)(3-e)$. The interaction time between particle motion and gas fluctuations, $t_{12}^{t}=t_{1}^{t}\left(1+C_{\beta} \zeta_{r}\right)^{-1 / 2}$, where $\zeta_{r}=3\left|U_{r}\right|^{2} / 2 k$ and $C_{\beta}$ is a constant.

Because the particulate stress depends on the granular temperature, we also need a closure equation for the balance of the fluctuational energy: 


$$
\frac{3}{2} \varepsilon_{2} \rho_{2} \frac{D}{D t} T=-\nabla \cdot \boldsymbol{q}-\tau: \nabla \boldsymbol{U}-\beta_{0}\left(3 T-k_{12}\right)+I
$$

The expression for the fluctuational energy flux, $\boldsymbol{q}$, is given as in Peirano and Leckner (1998), viz.

$$
\boldsymbol{q}=-\frac{3}{2} \varepsilon_{2} \rho_{2}\left(\kappa_{2}^{t}+\kappa_{2}^{c}\right) \nabla T
$$

The diffusion coefficients, $\kappa_{2}^{c}$ and $\kappa_{2}^{t}$, are

$$
\begin{aligned}
& \kappa_{2}^{c}=\varepsilon_{2} \chi(1+e)\left(\frac{6}{5} \kappa_{2}^{t}+\frac{4}{3} d \sqrt{\frac{T}{\pi}}\right), \\
& \kappa_{2}^{t}=\left(\frac{3 t_{12}^{t}}{5 t_{12}^{x}} k_{12}+\left(1+\varepsilon_{2} \chi C\right) T\right) /\left(\frac{9}{5 t_{12}^{x}}+\frac{D}{t_{2}^{c}}\right),
\end{aligned}
$$

where $C=3(1+e)^{2}(2 e-1) / 5$ and $D=(1+e)(49-33 e) / 100$. Energy dissipation is described by

$$
I=12 \varepsilon_{2}^{2}\left(e^{2}-1\right) \chi \rho_{2} \frac{1}{d \sqrt{\pi}} T^{\frac{3}{2}} .
$$

So far, no thorough numerical testing of this model has been accomplished. This generalized kinetic theory model of the particle phase in a gas-solid two-phase system needs to be explored numerically in order to evaluate the impact of gas turbulence on the viscosity and diffusion coefficients. We find below (figures 17 and 18) significant improvement on previous models which only used the kinetic theory of dry granular flow.

\section{Fully-developed steady flow}

Our new model will be tested on relatively dilute flows of high-inertia particles. To summarize the main features of our model: we have introduced a new description of the turbulent length scale which addresses the influence both of particles and the transport effect - this is given by equations (13), (19). The effect of particles on the turbulence model 
parameter $c_{3}$, is proposed in equation (20). Moreover, the recent generalized kinetic theory model of Peirano and Leckner (1998) is adopted for the particulate phase, which avoids the physical inappropriateness of previous models based on the kinetic theory of dry granular flow. The new transport equation for turbulent kinetic energy proposed by Crowe and his colleagues has also been incorporated. In addition, our recent work on inter-phase momentum and kinetic energy interactions, i.e. $\boldsymbol{F}_{d r a g}$ and $k_{12}$ respectively, is also used in the present model. Together, this new model represents a unique two-fluid approach to gas-solid flows and is the most comprehensive to date - incorporating improved physicality above that of previously-published models.

For a fully-developed, steady, axisymmetric, relatively dilute gas-solid flow in a vertical pipe, the governing equations then become,

\section{Solid phase}

- Momentum equation

$$
\begin{array}{ll}
-\varepsilon_{2} \frac{\partial P}{\partial z}-\frac{1}{r} \frac{\partial}{\partial r}\left(r \tau_{2 r z}\right)+\boldsymbol{F}_{d r a g, z}-\varepsilon_{2} \rho_{2} \boldsymbol{g}_{z}=0, & \text { axial direction } \\
\frac{1}{r} \frac{\partial}{\partial r}\left(r \tau_{2 r r}\right)-\frac{\tau_{2 \theta \theta}}{r}=0 . & \text { radial direction }
\end{array}
$$

- Fluctuational energy equation

$$
-\frac{1}{r} \frac{\partial}{\partial r}\left(r \boldsymbol{q}_{r}\right)-\tau_{2 r z} \frac{\partial u}{\partial r}-\beta_{0}\left(3 T-k_{12}\right)-I=0 .
$$

\section{Gas phase}

- Momentum equation

$$
-\varepsilon_{1} \frac{\partial P}{\partial z}+\frac{1}{r} \frac{\partial}{\partial r}\left[r\left(\mu+\mu_{t}\right) \frac{\partial v}{\partial r}\right]-\boldsymbol{F}_{d r a g, z}=0 . \quad \text { axial direction }
$$

- Turbulent kinetic energy equation

$$
\frac{1}{r} \frac{\partial}{\partial r}\left[\varepsilon_{1}\left(\mu+\frac{\mu_{t}}{\sigma_{k}}\right) r \frac{\partial k}{\partial r}\right]-\varepsilon_{1} \tau_{1 r z} \frac{1}{r} \frac{\partial}{\partial r}(r v)+\beta_{0}|u-v|^{2}+\beta_{0}\left(3 T-k_{12}\right)-\varepsilon_{1} \rho_{1} \varepsilon=0
$$


- Turbulent length scale

$$
\begin{aligned}
& \frac{1}{r} \frac{\partial}{\partial r}\left\{r \varepsilon_{1}\left(\mu+\frac{\mu_{t}}{\sigma_{\varepsilon}}\right) \frac{\partial \varepsilon}{\partial r}\right\}+c_{1} \varepsilon_{1} \frac{\varepsilon}{k} \mu_{t}\left(\frac{\partial V}{\partial r}\right)^{2}-c_{2} \rho_{1} \varepsilon_{1} \frac{\varepsilon^{2}}{k}+ \\
& \varepsilon=\frac{c_{\varepsilon 3} \beta_{0}\left[\mathbf{U}-\left.\mathbf{V}\right|^{2}+\left(3 T-k_{12}\right)\right] \frac{\varepsilon}{k}=0,}{1-\left(6 \varepsilon_{2} / \pi \varepsilon_{2 m}\right)^{1 / 3}} \frac{k^{3 / 2}}{l},
\end{aligned}
$$

where $u$ and $v$ represent averaged axial velocities of the particulate and gas phases, respectively, and subscripts $r$ and $z$ represent radial and axial components, respectively.

\section{Boundary conditions}

For a gas-solid flow, the particle diameter may not be negligible when compared to the width of the gas viscous boundary layer. Therefore, when we set the boundary conditions at the wall for the solid phase, we establish a momentum and energy balance at a thin layer of particles, the thickness of which may be the same order as that of the gas viscous layer or larger. The gas phase boundary condition should also be set on the same thin layer, as suggested by Sinclair and Jackson (1989). In addition, the derivation of governing equation (2) is based on an element volume containing statistically sufficient number of particles, so the length scale of that element volume must be at least the same order as the particle diameter. As a result, it is not appropriate to solve the governing equations from the pipe center right up to the wall, where a non-slip boundary condition for the gas phase would normally be set. Therefore, a high Reynolds number $k$ - $\varepsilon$ model is used here with the wall functions to determine the boundary values for the gas phase.

The wall functions for a single-phase flow are valid for flows with small particle volume fractions (Kulick et al., 1994). Therefore, we adopt von Karman's logarithmic law, and the magnitude of the slip velocity of gas at the wall is obtained as (Mohammadi and Pironneau, 1994), 


$$
v=\left(\frac{1}{K} \log y^{+}+5.5\right) \widetilde{v}, \quad 20 \leq y^{+} \leq 100,
$$

where, $\widetilde{v}=\sqrt{\tau_{1 s} / \rho_{1}}, y^{+}=y \widetilde{v} \rho_{1} / \mu, \tau_{1 \mathrm{~s}}$ is the gas shear stress at the wall and $y$ is the distance to the wall; $K$ is the von Karman constant $(K=0.41)$. If a local equilibrium can be assumed, i.e. the generation and dissipation of the turbulent kinetic energy is nearly the same, the gas turbulent kinetic energy, $k$, at the near wall region, can be described by (Louge et al., 1991; Ferziger and Perić, 2002):

$$
\frac{\partial k}{\partial r}=0
$$

The boundary condition for $\varepsilon$ can be derived by assuming a kinetic energy balance in the near-wall region:

$$
\left.\varepsilon\right|_{\text {wall }}=\frac{\left.C_{\mu}^{3 / 4} k\right|_{\text {wall }} ^{3 / 2}}{K y} .
$$

The above boundary conditions for the gas phase at the wall region are valid when the first grid point is within the logarithmic region, i.e. $y^{+} \geq 20$.

The collisions between particles and the wall play a significant role in the flow in small-diameter pipes, especially for high-inertia particles which do not respond quickly to the gas flow. Currently, there are two main competing boundary conditions for the solid phase, i.e. those due to Johnson et al. (1990) and Jenkins and Louge (1997).

Jenkins and Louge (1997) built on the work of Jenkins (1992) by rigorously deriving boundary conditions for the stress and fluctuational energy for particle flow over a plane frictional wall. Assuming a Maxwellian velocity distribution, they integrated the impulse equations for the particle-wall collision. As their derived conditions are empirical-parameter free, it is clear that their work is a major step to a mature advanced theory for the boundary conditions. However, their boundary conditions also have some shortcomings. A particlewall collision will be sliding if 


$$
\left|u_{t}-\frac{1}{2} d \omega\right|>\frac{7}{2} \mu_{s}\left(1+e_{w}\right) u_{n}
$$

where $u_{t}$ and $u_{n}$ are the particle translational velocities parallel and normal to the wall, $e_{w}$ is the particle-wall coefficient of restitution, $\mu_{s}$ is the static coefficient of friction, and $\omega$ is the particle angular velocity. Only in the limit of all-sliding collisions can a rigorous expression for the stress and fluctuational energy flux be derived. However, in real situations, both sliding and non-sliding collisions generally occur. As has been stated, Jenkins and Louge assumed a Maxwellian velocity distribution, so some of the collisions must be sliding and others non-sliding for a finite small $\mu_{s}$ — the all-sliding limit can only occur when $\mu_{s}$ is zero. A discontinuity therefore arises in the integral. They also, incidentally, ignore fluctuations in the angular velocity.

Furthermore, although these boundary conditions are analytically-derived, they are not without parameters which, in practice, have to be optimized. As Sommerfeld (1992) stated, the parameters which affect a particle-wall collision are the particle collision angle, the particle translational and rotational velocity before collision, the properties of the particle and the wall materials, the particle shape, and the roughness of the wall surface. Of these, the particle-wall normal coefficient of restitution is strongly velocity-dependent. Also, the coefficient of friction and tangential coefficient of restitution are collision geometrydependent and, for a small-diameter pipe, the pattern of particle-wall collisions is likely to differ from that for a plane wall. With these difficulties in properly characterizing the collisions, as well as the discontinuity in the integral outlined above, the complications arising in the necessary averaging process are likely to be substantial. Even if these coefficients (particle-wall normal and tangential coefficient of restitution, and coefficient of friction) can be assumed as constants, they are hardly measurable. In spite of their clear physical meaning, these three parameters have to be tuned in practice. 
The boundary conditions of Johnson et al. (1990) adopt an alternative approach. Although not mathematically rigorous, employing a single specularity coefficient, $\phi$, (which is an empirical "tunable" constant) admits flexibility to the boundary conditions, so the averaged influences of the geometry of the curved pipe wall and the variety of particle-wall collisions can be encapsulated under one parameter. Despite the profile of granular temperature being sensitive to this parameter (as shown in figures 22 and 23 below), we can ensure comparability of our numerical simulation results by adjusting the specularity coefficient for one operational condition then maintaining this singular value for all our subsequent simulations. Although the boundary conditions of Johnson et al. are the most widely used and tested so far for gas-solid flow modeling, more sophisticated boundary conditions are still needed for complex and realistic boundary geometries.

Here, we summarize the boundary conditions of Johnson et al. (1990). The shear stress generated on the thin flow layer (i.e. the control volume) above the wall is

$$
\tau_{2 r z}=\omega \phi \rho_{2} \varepsilon_{2} u \sqrt{3 T} \chi
$$

The energy dissipation term is

$$
D=\frac{\alpha}{2} \rho_{2} \varepsilon_{2}(3 T)^{\frac{3}{2}}\left(1-e_{w}^{2}\right) \chi
$$

where $\alpha$ and $\omega$ are dimensionless proportionality constants of order unity which are given by

$$
\alpha=\omega=\frac{\pi}{6 v_{2 m}}
$$

The balance of energy requires,

$$
u \tau_{r z}-D=q_{r}
$$

In the centerline of the pipe, axisymmetric conditions are applied, i.e.

$$
\frac{\partial u}{\partial r}=0, \frac{\partial v}{\partial r}=0, \frac{\partial \varepsilon_{2}}{\partial r}=0, \frac{\partial T}{\partial r}=0, \frac{\partial k}{\partial r}=0, \frac{\partial \varepsilon}{\partial r}=0
$$




\section{Results and discussion}

In this section we first examine the predictions of our new model. Then we investigate the relative importance of the new model components we have introduced, and perform a sensitivity analysis on our model parameters.

Non-intrusive laser Doppler measurements of fully-developed turbulent gas-solid flows in a vertical pipe have been reported by Maeda et al. (1980), Lee and Durst (1982) and Tsuji et al. (1984). Glass particles with a density of $2590 \mathrm{kgm}^{-3}$, in glass pipes with internal diameters $56 \mathrm{~mm}$ and $41.8 \mathrm{~mm}$, were used by Maeda et al. and Lee and Durst, respectively. The experimental data produced by Tsuji et al. is more comprehensive, involving differentlysized particles, different mass loading ratios and various superficial gas velocities. Latterly, Tsuji revisited these data and experimental measurements of the axial fluctuations of the particulate phase were published in the work of Bolio et al. (1995). Tsuji et al. used polystyrene spheres with a density of $1020 \mathrm{kgm}^{-3}$ in a pipe with an internal diameter of 30.5 $\mathrm{mm}$. The ratio of particle-to-gas mass flow rates, i.e. mass loading, $m$, is up to 4.2 .

The particle-particle and particle-wall collisions are often the major source of particulate stresses, but the coefficients of restitution, $e$ and $e_{w}$ respectively, depend on the impact velocity and are extremely sensitive parameters. However, reasonably-estimated constant values of $e$ and $e_{w}$ can give at least a first order prediction when the collisions are nearly elastic in a relatively dilute flow, because in this case the collisions do not contribute significantly to the particulate stresses and, as we show below, our simulation results do not strongly depend on the coefficients of restitution. Therefore we treat the coefficients of restitution as constants within the scope of this paper, with $e$ and $e_{w}$ for the cases of Maeda et al. (1980) and Lee and Durst (1982) both taken as 0.94 (Bolio et al. 1995). We adopt $e=0.9$ and $e_{w}=0.75$ for the case of Tsuji et al. (1984), as discussed in Louge et al. (1991) and Bolio et al. (1995). 
Finally, we take the gas density to be $1.2 \mathrm{kgm}^{-3}$, the specular parameter in the boundary conditions, $\phi$, to be 0.008 , and the turbulence parameter, $c_{3}$, in our expression for $c_{\varepsilon 3}$ to be 1.95 . These singular values of $\phi$ and $c_{3}$ are obtained once by optimizing to produce good agreement with the single set of measurements of Tsuji et al. (1984) for a mass loading ratio, $m=3.2$. They are then kept the same for all the other cases, including Maeda et al. (1980) and Lee and Durst (1982). A summary of these parameters is given in Table 1. Bolio et al. (1995) chose a smaller value for $\phi$, i.e. 0.002: this difference may stem from the different turbulence modulation models used and the lack of any damping effect of the interstitial gas on the particulate stresses.

For fully-developed steady flow in an axi-symmetric pipe, the governing equations reduce to the five coupled second-order, non-linear ordinary differential equations and two algebraic equations, given in equations (31)-(37). Because the achievement of numerical convergence is very sensitive to the initial guess, the equations are decoupled and linearized. Finite differences are then used to solve these ordinary differential equations, and convergence is rapidly achieved by using an over-relaxation method.

The first point to note in our numerical results for dilute gas-solid flows is that the predicted particle radial segregation is negligible (see figure 2), which agrees with the experimental observations of Tsuji et al. (1984), and the simulations of Louge et al. (1991). Because a high Reynolds number $k-\varepsilon$ model with wall functions is used the solutions do not include the viscous sublayer.

In figures 3-5, the effect of particles on the gas mean velocity can be distinguished. Our simulation results are in reasonable agreement with experimental data for the gas velocity profiles. Generally, the presence of particles leads to a flatter gas velocity profile. Maeda et al. (1980), Lee and Durst (1982) and Tsuji et al. (1984) all observed that the particle mean velocity near the wall can be larger than the gas mean velocity. This effect is captured 
by the present model, and can be understood in terms of the particulate shear stress (Louge et al., 1991). Comparing the velocity profiles of figures 4 and 5, it can be seen that the location where the particle velocity is predicted to be above the gas velocity is closer to the pipe center for smaller particles. This is also confirmed by Tsuji et al. (1984).

The effect of mass loading on the mean velocity field of both phases is shown in figures 6 and 7. In figure 6 , the predictions clearly show the gas velocity profile becomes flatter with increasing mass loading, which has been experimentally observed by Tsuji et al. (1984). Simulation results shown in figure 7 predict the tendency for a smaller mean slip velocity between the two phases with increased mass loading, which is in agreement with experimental observation. However, our predicted particle velocity profiles are flatter than the measured ones, which is perhaps due to our assumption of uniform particle size. In real situations there is a particle size distribution: smaller particles respond to the gas flow at the pipe center more easily and larger ones are more likely to lag behind in the region near the wall (Mathiesen et al., 2000).

Figures 8-13 show the impact of inter-phase interactions on the fluctuations of both phases. The effect of particles on gas fluctuations is shown in figures 8-11 and 13 under different mass loadings and different particle sizes. The gas turbulent energy profiles for 200 $\mu \mathrm{m}$ diameter particles at different mass loadings can be seen in figures 8,9 and 11 . In comparison to the corresponding pure gas flow, the particles attenuate the gas turbulence. The $500 \mu \mathrm{m}$ diameter particles augment the gas turbulence as can be seen in figure 10 , especially at the pipe center, and the greater the mass loading the more the gas turbulence is enhanced. Particles of all sizes examined cause the profiles of the gas turbulent energy to flatten. Different sized particles have substantially different effects on the turbulence, as seen in figure 13 comparing particle-laden and particle-free flows under the operating conditions of Lee and Durst (1982). The attenuation associated with $200 \mu \mathrm{m}$ diameter particles, and the 
enhancement associated with $500 \mu \mathrm{m}$ particles, is consistent with the experimental observations. This confirms the general conclusion given by Gore and Crowe (1989) that smaller particles will attenuate turbulence while larger ones enhance it. As with previous models, e.g. Louge et al. (1991) and Bolio et al. (1995) where they predicted the general trend of the turbulence modulation, the quantitative discrepancies with experimental data are still large. This may be due to the anisotropic nature of turbulence in a real pipe flow (Sinclair and Mallo, 1998).

Figures 8, 9 and 12 show comparisons of the measured and predicted r.m.s axial velocity fluctuations of particulate phase. The fluctuations of the particulate phase are larger than those of the gas phase, especially at small mass loading, as seen in figure 9. Increasing mass loading will attenuate the fluctuations, which can be understood in terms of "the free path" of the particle motion. From these figures, we can see the fluctuations are overpredicted for $m=4.2$ and underestimated for $m=1.3,1.0$. As shown in figures 22 and 23 later, the specularity coefficient $\phi$ has an effect on the particle fluctuations. Bolio and Sinclair (1995) also showed the same trend. Therefore, this result may indicate that, under the formulation of this model, the specularity parameter may need to incorporate the effect of solid volume fraction.

Having shown the general utility of our new model, we now turn to examine the relative importance of its constituent parts. Figures 14-16 compare the various turbulent kinetic energy source and sink terms in order to determine the most important factor in our model which influences turbulence modulation. Figure 14 compares the radial variation of the turbulent energy additional source terms due to the presence of particles, and the viscous dissipation term in equation (35). Except in the wall region, it can be seen that the turbulent energy in the pipe is mainly generated from the work done by the drag force, i.e. $\beta_{0}|\boldsymbol{U}-\boldsymbol{V}|^{2}$. Because turbulent energy generated by a velocity gradient is dominant in the wall region, the 
turbulent energy dissipation, $\varepsilon_{1} \rho_{1} \varepsilon$, is larger than $\delta k$, which is $\beta_{0}|\boldsymbol{U}-\boldsymbol{V}|^{2}+\beta_{0}\left(3 T-k_{12}\right)$. This additional energy source, i.e. $\beta_{0}|\boldsymbol{U}-\boldsymbol{V}|^{2}$, is the major difference between Crowe's new model and previous commonly-used models. Figures 15 and 16 also confirm that the source term due to interaction with the particles, rather than velocity gradient, contributes more to the turbulence, except in the wall region where the contribution from the velocity gradient becomes more important.

Due to the complex nature of gas-solid flows, only a complete investigation of every individual model component can distinguish the importance of the competing mechanisms involved. The objectives of figures 17 and 18 are: i) to determine whether inter-particle collisions or particle random fluctuational motion contributes most to the particulate viscosity, $\psi_{2}$, and the diffusion coefficient, $\kappa_{2}$ in these relatively dilute flows; ii) to determine the impact of gas turbulence on the particulate viscosity, $\psi_{2}$, and the diffusion coefficient, $\kappa_{2}$. Here, if the interstitial gas is ignored, as in the standard kinetic theory of dry granular flow, the results are denoted as "without gas"; otherwise as "with gas". First, we see in these figures that the collisional contribution to the particulate viscosity, $\psi_{2}$, and the diffusion coefficient, $\kappa_{2}$, is very small. Therefore, the turbulent components, $\psi_{2}^{t}$, and $\kappa_{2}^{t}$, are dominating for these relatively dilute flows. Second, the effect of the turbulent gas on the particulate viscosity and diffusion coefficient is very significant. The interstitial gas plays a damping role which reduces both the particulate viscosity and the diffusion coefficient. The reduction of viscosity will lead to a decrease in particulate stresses. Therefore, interactions between the two phases play a more important role, while inter-particle collisions are less influential in these relatively dilute flows. As a result, ignoring the gas turbulence effect on the constitutive equations for the particulate phase, as previous models have done, 
significantly over-predicts $\psi_{2}$ and $\kappa_{2}$, and is not appropriate for these flows. Only in a dense flow may the particulate stresses relating to the interstitial gas be regarded as negligible.

Because any turbulence model inevitably introduces some empiricism, a thorough test and validation of these parameters is essential to any two-fluid model. A sensitivity analysis of our model to these parameters is shown in figures 19-26. The effect of the particle-particle coefficient of restitution on the axial velocity fluctuations of both phases is shown in figures 19-21. We see that this coefficient has a negligible effect on the gas turbulence but has a greater influence on the granular temperature, for a mass loading ratio, $m=3.2$. For the smaller mass loading ratio, $m=1.0$, shown in figure 21 , we see the effect of the coefficient of restitution on the granular temperature is less. Generally, the sensitivity of solutions to the coefficient of restitution, which has been observed in dense flows, is not significant for a relatively dilute flow. This result lends support to our earlier assumption of a constant coefficient of restitution.

Figures 22 and 23 show the effect of the specularity coefficient on the fluctuations of both phases. The impact of this parameter is relatively insignificant to the gas phase but significant to the particulate phase. Increasing the value of the specularity coefficient is found to increase particle fluctuations. The turbulence model constant, $c_{3}$, used in our expression for $c_{\varepsilon 3}$, is examined in figures 24 and 25 . This parameter has a negligibly small influence on fluctuations of the particulate phase but has significant effect on gas fluctuations. If we turn off the modification on $c_{3}$ and maintain a constant value of $c_{3}=1.95$, the prediction of the gas turbulent kinetic energy profile is found to be at least one order of magnitude smaller. The most important influence on the fluctuational motion of the gas and particle phases is the inter-particle length scale, which is addressed via equation (18). In figure 26, we can see that even the small difference of not including the normalization with $\varepsilon_{2 m}$ in equation (18) leads to very different predictions of the gas turbulent kinetic energy. Furthermore, if we turn off this 
modification entirely (which means that the inter-particle space effect on turbulence length scale is then excluded) we find a major discrepancy in the calculation for the turbulent kinetic energy profile, as expected. Therefore, it is most important to assess accurately how the interparticle length scale can be incorporated into a turbulent model. Our results are consistent with the general observation that for a solid volume fraction even as low as $10^{-6}$ the particles will affect the gas turbulence (Elghobashi, 1994). The present model is a practical attempt at tackling the effect of the inter-particle length scale.

\section{Summary and conclusions}

We have proposed a new model for gas turbulence modulation in gas-solid flows, and examined the interstitial gas influence on the particulate stresses. Our simulation results are generally in reasonable quantitative, and good qualitative, agreement with published experimental data. We may draw the following conclusions:

- The inter-particle length scale has a significant effect on the gas turbulence, and this should be incorporated in a turbulence model.

- The work done due to the drag force is responsible for enhancing the gas turbulence.

- The carrier gas turbulence plays an important role in determining the particulate stresses, so that ignoring the carrier gas in the constitutive equations for the particulate phase, as previous models have done, is not appropriate for relatively dilute gas-solid flows.

- The effect of particles on turbulence parameters needs to be evaluated and tested in order to facilitate gas-solid system simulation and design.

The present turbulence modulation model is based on an isotropic assumption, which is its most significant limitation. Sheen et al. (1993) have demonstrated the anisotropic nature of gas turbulence in pipe flow that needs to be addressed in a future more sophisticated model. 


\section{Acknowledgement}

The authors are grateful to the referees and Professor S. Sundaresan for their valuable suggestions which have helped to improve this paper. The work was carried out under the sponsorship of the EPSRC of the United Kingdom through grant no. GR/M33617. 


\section{Literature Cited}

Abu-Zaid, S., and G. Ahmadi, "Simple kinetic model for rapid granular flows including frictional losses," J. Eng. Mech. ASCE, 116, 379 (1990).

Anderson, T. B., and R. Jackson, "Fluid mechanical description of fluidized beds: comparison with theory and experiment," I \& EC Fund. 6, 527 (1967).

Agrawal, K, P. N. Loezos, M. Syamlal, and S. Sundaresan, "The role of meso-scale structures in rapid gas-solid flows," J. Fluid Mech., 445, 151 (2001).

Bagnold, R. A., "Experiments on a gravity-free dispersion of large solid particles in a Newtonian fluid under shear," Proc. R. Soc. London A 225, 49 (1954).

Balzer, G., A. Boelle, and O. Simonin, “ Eulerian gas-solid flow modeling of dense fluidized beds," In Fluidization VIII, 1125 (1995).

Berlemont, A., P. Desjonqueres, and G. Gouesbet, "Particle lagrangian simulation in turbulent flows," Int. J. Multiphase Flow 16, 19 (1990).

Boelle, A., G. Balzer, and O. Simonin, "Gas-solid flows," In Gas-solid flows. ASME FED, 228, 9, (1995).

Bolio, E. J., and J. L. Sinclair, "Gas turbulence modulation in the pneumatic conveying of massive particles in vertical tubes," Int. J. Multiphase Flow 21, 985 (1995).

Bolio, E., J. Yasuna, and J. Sinclair, "Dilute turbulent gas-solid flow in risers with particle interactions," AIChE J. 41, 1375 (1995).

Buyevich, Y.A., "Particulate stresses in dense disperse flow," Ind. Eng. Chem. Res. 38, 731 (1999).

Cao, J., and G. Ahmadi, "Gas-particle two-phase turbulent flow in a vertical duct," Int. J. Multiphase Flow 21, 1203 (1995).

Chen, P. E., and C. E. Wood, "A turbulence closure model for dilute gas-particle flows," Can. J. Chem. Eng. 63, 349 (1985). 
Crowe, C. T., "Recent advances in modelling of turbulent gas particle flows," ISMNP' 97, Int. Symp. Multiphase Fluid, Non-Newtonian Fluid and Physico Chemical Fluid Flows. (1997).

Crowe, C. T., and I. Gillandt, "Turbulence modulation of fluid-particle flows - a basic approach," Third International Conference on Multiphase Flows, ICMF'98, Lyon, France (1998).

Crowe, C. T., "On models for turbulence modulation in fluid-particle flows," Int. J. Multiphase Flow 26, 719 (2000).

Crowe, C. T., and P. Wang, "Towards a universal model for carrier-phase turbulence in dispersed phase flows," Proc. of 2000 ASME FED Summer Meeting, FEDSM200011132, Boston, USA (2000).

Dasgupta, S., R. Jackson, and S. Sundaresan, "Turbulent gas-particle flow in vertical risers," AIChE J. 40, 215 (1994).

Dasgupta, S., R. Jackson, and S. Sundaresan, "Gas-particle flow in vertical pipes with high mass loading of particles," Powder Tech. 96, 6 (1998).

Di Felice, R., "The voidage function for fluid-particle interaction systems," Int. J. Multiphase Flow 20, 153 (1994).

Elghobashi, S. E., “On predicting particle-laden turbulent flows,” Appl. Sci. Reserch 52, 309 (1994).

Ferziger, J. H. and M. Perić, Computational methods for fluid dynamics, Springer, New York (2002).

Fessler, J. R., and J. K. Eaton, “Turbulence modification by particles in a backward-facing step flow," J. Fluid Mech. 394, 97 (1999).

Gidaspow, D., Multiphase flow and fluidization, Academic Press, London (1994).

Goldhirsch I., and G. Zanetti “Clustering instability in dissipative gases," Phys. Rev. Lett. 70, 1619 (1993). 
Gore, R. A., and C. T. Crowe, "Effect of particle size on modulating turbulent intensity," Int. J. Multiphase Flow 15, 279 (1989).

Hardalupas, Y., A. M. K. P., Taylor, and J. H. Whitelaw, "Velocity and particle-flux characteristics of turbulent particle-laden jets," Proc. R. Soc. London A 426, 31 (1989).

Hrenya, C., E. Bolio, D. Chakrabarti, and J. Sinclair, "Comparative study of low Reynolds k$\varepsilon$ models in predicting turbulent pipe flow," Chem. Eng. Sci. 50, 1923 (1995).

Jackson, R., "Locally averaged equations of motion for a mixture of identical spherical particles and a Newtonian fluid," Chem. Eng. Sci. 52, 2457 (1997).

Jenkins, J. T., "Boundary conditions for rapid granular flow: flat, frictional walls," J. Applied Mech. 59, 120 (1992).

Jenkins, J. T., and M. Y. Louge, “On the flux of fluctuation energy in a collisional grain flow at a flat, frictional wall," Phys. Fluids 9, 2835 (1997).

Jenkins, J. T., and M. W. Richman, “Grad's 13-moment system for a dense gas of inelastic spheres," Arch. Ration. Mech. Anal. 87, 3485 (1985).

Jenkins, J. T., and S. B. Savage, "A theory for the rapid flow of identical, smooth, nearly elastic particles," J. Fluid Mech. 130, 187 (1983).

Johnson, P. C., P. Nott, and R. Jackson, "Frictional-collisional equations of motion for particulate flows and their application to chutes," J. Fluid Mech. 210, 501 (1990).

Kadanoff, L. P., "Built upon sand: theoretical ideas inspired by granular flows," Rev. Mod. Phys. 71, 435 (1999).

Kenning, V. M., and C. T. Crowe, "Effect of particle on carrier phase turbulence in gasparticle flows," Int. J. Multiphase Flow 23, 403 (1997).

Koch, D., "Kinetic theory for a monodisperse gas-solid suspension," Phys. Fluids A, 2, 1711 (1990). 
Koch, D., and A. S. Sangani, "Particle pressure and marginal stability limits for a homogeneous monodisperse gas-fluidized bed: kinetic theory and numerical simulations," J. Fluid Mech. 400, 229 (1999).

Kovacs, A. E., "Prandtl's mixing length concept modified for equilibrium sendiment-laden flows," J. Hydraulic Eng. 124, 803 (1998).

Kulick, J. D., J. R. Fessler, and J. K. Eaton, "Particle response and turbulence modification in fully developed channel flow," J Fluid Mech. 277, 109 (1994).

Lee, S. L., and F. Durst, "On the motion of particles in turbulent duct flows," Int. J. Multiphase Flow 8, 125 (1982).

Louge, M. Y., E. Mastorakos, and J. K. Jenkins, “The role of particle collisions in pneumatic transport," J. Fluid Mech. 231, 345 (1991).

Lun, C. K. K., and S. B. Savage, "A simple kinetic theory for granular flow of rough, inelastic, spherical particles," Trans. ASME J. Appl. Mech. 54, 47 (1987).

Lun, C. K. K., S. B. Savage, D. J Jeffrey, and N. Chepurniy, "Kinetic theory for granular flow: inelastic particles in Couette flow and slightly inelastic particles in a general flowfield," J. Fluid Mech. 140, 223 (1984).

Ma, D., and G. Ahmadi, “A kinetic model for rapid granular flows of nearly elastic particles including interstitial fluid effects," Powder Tech. 56, 191 (1988).

Maeda, M., K. Hishida, and T. Furutani, “Optical measurements of local gas and particle velocity in an upward flowing dilute gas-solid suspension," Polyphase Flow and Transport Technology, 211, Century 2-ETC, San Francisco (1980).

Mathiesen, V., T. Solberg, and B. H. Hjertager, "Predictions of gas/particle flow with an Eulerian model including a realistic particle size distribution," Powder Tech. 112, 34 (2000). 
Michelassi, V., W. Rodi, and J. Zhu, "Testing a low Reynolds number $k$ - $\varepsilon$ turbulence model based on direct numerical simulation," AIAA J. 31, 1720 (1993).

Mohammadi, B., and O. Pironneau, Analysis of the k-epsilon turbulence model, John Wiley \& Sons, New York (1994).

Mostafa, A. A., and H. C. Mongia, "On the interaction of particles and turbulent fluid flow," Int. J. Heat Mass Transfer 31, 2063 (1988).

Ogawa, S., A. Umemura, and N. Oshima, "On the equations of fully fluidized granular materials," J. Appl. Math. Phys. (ZAMP) 31, 483 (1980).

Peirano, E., and B. Leckner, "Fundamentals of turbulent gas-solid flows applied to circulating fluidized bed combustion," Prog. Energy Combust. Sci. 24, 259 (1998).

Pita, J., and S. Sundaresan, "Gas-solid flow in vertical tubes," AIChE J. 37, 1009 (1991).

Rizk, M., and S. Elghobashi, “A two-equation turbulence model for dispersed dilute confined two-phase flows," Int. J. Multiphase Flow. 15, 119 (1989).

Rodi, W., and N. N. Mansour, "Low Reynolds number $k-\varepsilon$ modelling with the aid of direct numerical simulation," J. Fluid Mech. 250, 509 (1993).

Sato Y., V. Fukuichi, and K. Hishida, "Effect of inter-particle spacing on turbulence modulation by Lagrangian PIV," Int. J. Heat Fluid Flow 21, 554 (2000).

Savage, S.B., and D. J. Jeffrey, "The stress tensor in a granular flow at high shear rates," $J$. Fluid Mech. 110, 255 (1981).

Sheen, H. J., Y. Z. Chang, and Y. S. Chiang, "Two-dimensional measurements of flow structure in a two-phase vertical pipe flow," Proc. Natl. Sci. Counc. ROC(A), 17, 200 (1993).

Sinclair, J. L., and R. Jackson, "Gas-particle flow in a vertical pipe with particle-particle interactions," AIChE J. 35, 1473 (1989). 
Sinclair, J. L., and T. Mallo, "Describing particle-turbulence interaction in a two-fluid modeling framework," Proc. ASME FEDSM'98, FEDSM98-5018, Washington, D. C., USA (1998).

Sommerfeld, M., "Modeling of particle-wall collisions in confined gas-particle flows, " Int. J. Multiphase Flow 18, 905 (1992).

Squires, K. D., and J. K. Eaton, "Effect of selective modification of turbulence on twoequation models for particle-laden turbulent flows," J. Fluids Eng. 116, 778 (1994).

Thai Van, D., J. Minier, O. Simonin, P. Freydier, and J. Olive, "Multidimensional two-fluid model computation of turbulent dispersed two-phase flows," ASME, Numerical Methods in Multiphase Flows, FED-185, 277 (1994).

Tsuji, Y., Y. Morikawa, and H. Shiomi, "LDV measurements of an air-solid two-phase flow in a vertical pipe," J. Fluid Mech. 139, 417 (1984).

Wen, C. Y., and Y. H. Yu, "Mechanics of fluidization," AIChE Symp. Ser. 62, 10 (1966).

Yarin, L. P., and G. Hetsroni, "Turbulence intensity in dilute two-phase flows," Int. J. Multiphase Flow 20, 27 (1994).

Yuan, Z., and E. Michaelides, “Turbulence modulation in particulate flows - a theoretical approach," Int. J. Multiphase Flow 18, 779 (1992).

Zaichik, L. I., V. A. Pershukov, M. V. Kozelev, and A. A. Vinberg, "Modeling of dynamics, heat transfer, and combustion in two-phase turbulent flows: 1. isothermal flows," Exp. Thermal Fluid Sci. 15, 291 (1997).

Zhang, Y.H., and J. M. Reese, "Particle-gas turbulence interactions in a kinetic theory approach to granular flows," Int. J. Multiphase Flow 27, 1945 (2001).

Zhang, Y.H., and J. M. Reese, "The drag force in two-fluid models of gas-solid flows," Chem. Eng. Sci. 58, 1641 (2003). 
Table 1. The parameters used in the simulations

\begin{tabular}{|c|c|c|c|}
\hline Parameters & Tsuji et al. (1984) & Maeda et al. (1980) & Lee and Durst (1982) \\
\hline Pipe diameter, $\mathrm{mm}$ & 30.5 & 56 & 41.8 \\
\hline Particle diameters, $\mu \mathrm{m}$ & 200,500 & 136 & 100,200 \\
\hline Particle density, $\mathrm{kgm}^{-3}$ & 1020 & 2590 & 2590 \\
\hline $\begin{array}{c}\text { Model constants, } C_{\mu}, c_{1} \text {, } \\
c_{2}, c_{3}, \sigma_{k}, \sigma_{\varepsilon}\end{array}$ & $\begin{array}{c}0.09,1.44,1.92 \\
1.95,1.3,1.3\end{array}$ & $\begin{array}{c}0.09,1.44,1.92 \\
1.95,1.3,1.3\end{array}$ & $\begin{array}{c}0.09,1.44,1.92 \\
1.95,1.3,1.3\end{array}$ \\
\hline $\begin{array}{l}\text { Inter-particle coefficient } \\
\text { of restitution, } e\end{array}$ & 0.9 & 0.94 & 0.94 \\
\hline $\begin{array}{l}\text { Particle-wall coefficient } \\
\text { of restitution, } e_{w}\end{array}$ & 0.75 & 0.94 & 0.94 \\
\hline Specularity coefficient, $\phi$ & 0.008 & 0.008 & 0.008 \\
\hline
\end{tabular}




\section{FIGURE CAPTIONS}

Figure 1. Radial variations of (a) gas normalized axial velocity, $v / v_{c}$, and (b) gas normalized r.m.s. fluctuational axial velocity, $v^{\prime} / v_{c}$, for a pure gas flow. The gas axial velocity at the pipe center, $v_{c}=13.4 \mathrm{~ms}^{-1}$. Other parameters as in Tsuji et al. (1984)

Figure 2. Typical radial variation of particle volume fraction, $\varepsilon_{2}$, in this case for $200 \mu \mathrm{m}$ diameter particles at a mass loading ratio, $m=1.0$. Other parameters as in Tsuji et al. (1984).

Figure 3. Radial variations of normalized axial velocity of both phases, $v / v_{c}$ and $u / v_{c}$. The mass loading ratio is, $m=0.3, \mathrm{Re}=22,000$ and the particles are $136 \mu \mathrm{m}$ diameter. Other parameters as in Maeda et al. (1980).

Figure 4. Radial variations of normalized axial velocity of the two phases, $v / v_{c}$ and $u / v_{c}$. The particles are $100 \mu \mathrm{m}$ diameter; the gas axial velocity at the pipe center, $v_{c}=5.7 \mathrm{~ms}^{-1}$; the mean solid volume fraction, $\varepsilon_{2}=5.8 \times 10^{-4}$; and other parameters as in Lee and Durst (1982).

Figure 5. Radial variations of normalized axial velocity of the two phases, $v / v_{c}$ and $u / v_{c}$. The particles are $200 \mu \mathrm{m}$ diameter; the gas axial velocity at the pipe center, $v_{c}=5.84 \mathrm{~ms}^{-1}$; the mean solid volume fraction, $\varepsilon_{2}=6.3 \times 10^{-4}$; and other parameters as in Lee and Durst (1982).

Figure 6. Radial variations of gas normalized axial velocity, $v / \nu_{c}$. The particles are $200 \mu \mathrm{m}$ diameter. The gas axial velocities at the pipe center, $v_{c}=10.8,11.9$ and $13.1 \mathrm{~ms}^{-1}$ for mass loading ratios, $m=3.2,1.9$ and 0.5 , respectively. Other parameters as in Tsuji et al. (1984).

Figure 7. Radial variations of normalized axial velocity of the particulate phase, $u / v_{c}$. The particles are $200 \mu \mathrm{m}$ diameter. The gas axial velocities at the pipe center, $v_{c}=14.6,17.4$ and 
$18.9 \mathrm{~ms}^{-1}$ for mass loading ratios, $m=4.2,2.1$ and 1.0 , respectively. Other parameters as in Tsuji et al. (1984).

Figure 8. Radial variations of normalized axial r.m.s fluctuational velocity of the two phases, $v^{\prime} / v_{c}$ and $u^{\prime} / v_{c}$. The particles are $200 \mu \mathrm{m}$ diameter. The gas axial velocity at the pipe center, $v_{c}=10.8 \mathrm{~ms}^{-1}$, and mass loading ratio, $m=3.2$. Other parameters as in Tsuji et al. (1984).

Figure 9. Radial variations of normalized axial r.m.s fluctuational velocity of the two phases, $v^{\prime} / v_{c}$ and $u^{\prime} / v_{c}$. The particles are $200 \mu \mathrm{m}$ diameter. The gas axial velocity at the pipe center, $v_{c}=12.8 \mathrm{~ms}^{-1}$, and mass loading ratio, $m=1.3$. Other parameters as in Tsuji et al. (1984).

Figure 10. Radial variations of gas normalized axial r.m.s fluctuational velocity, $v / v_{c}$. The particles are $500 \mu \mathrm{m}$ diameter. The gas axial velocities at the pipe center, $v_{c}=10.7,11.4$ and $13.3 \mathrm{~ms}^{-1}$ for mass loading ratios, $m=3.4,2.9$ and 1.3 respectively. Other parameters as in Tsuji et al. (1984).

Figure 11. Radial variations of gas normalized axial r.m.s fluctuational velocity, $v / v_{c}$. The particles are $200 \mu \mathrm{m}$ diameter. The gas axial velocities at the pipe center and mass loading ratios are (a) $v_{c}=11.9 \mathrm{~ms}^{-1}, m=1.9$; and (b) $v_{c}=12.8 \mathrm{~ms}^{-1}, m=0.9$. Other parameters as in Tsuji et al. (1984).

Figure 12. Radial variations of normalized axial r.m.s fluctuational velocity of the particulate phase, $u^{\prime} / v_{c}$. The particles are $200 \mu \mathrm{m}$ diameter. The gas axial velocities at the pipe center and mass loading ratios are (a) $v_{c}=14.6 \mathrm{~ms}^{-1}, m=4.2$; and (b) $v_{c}=18.9 \mathrm{~ms}^{-1}, m=1.0$. Other parameters as in Tsuji et al. (1984).

Figure 13. Simulation results for radial variations of gas normalized r.m.s. fluctuational axial velocity, $v^{\prime} / v_{c}$. The gas axial velocity at the pipe center, $v_{c}=5.84 \mathrm{~ms}^{-1}$. For particle-laden flow, 
the mass loading, $m=1.4$, and the particles are $200 \mu \mathrm{m}$ and $500 \mu \mathrm{m}$ diameter. Other parameters as in Lee and Durst (1982).

Figure 14. Comparison of radial variation of normalized turbulent energy source and sink terms: $\delta k_{1}=(\boldsymbol{U}-\boldsymbol{V})^{2} / k, \delta k_{2}=3 T / k, \delta k_{3}=-k_{12} / k$ and $\delta k_{4}=-\varepsilon_{1} \rho_{1} \varepsilon / \beta_{0} k$. The particle diameter is $200 \mu \mathrm{m}$ and mass loading ratio, $m=1.0$. Other parameters as in Tsuji et al. (1984).

Figure 15 Comparison of normalized turbulent main generation term, due to particles, $\delta k=|\boldsymbol{U}-\boldsymbol{V}|^{2} / k$ and the viscous dissipation term, $\delta \varepsilon=-\varepsilon_{1} \rho_{1} \varepsilon / \beta_{0} k:$ a) $m=1.0$ and b) $m=3.2$, for $200 \mu \mathrm{m}$ particles. Other parameters as in Tsuji et al. (1984).

Figure 16. Radial variation of the ratio of the sink, $\delta \varepsilon_{s}=c_{2} \varepsilon_{1} \rho_{1} \varepsilon^{2} / k$, to the source, $\delta \varepsilon_{g}=c_{\varepsilon 3} \beta_{0}\left[|\boldsymbol{U}-\boldsymbol{V}|^{2}+\left(3 T-k_{12}\right)\right] \varepsilon / k$, in equation (36), $\varepsilon^{*}=\delta \varepsilon_{s} / \delta \varepsilon_{g}$. Particle diameter is $200 \mu \mathrm{m}$ and mass loading ratio, $m=3.2$. Other parameters as in Tsuji et al. (1984).

Figure 17. Comparison of radial variations of particulate viscosity components: a) turbulent (superscript t) and b) collisional (superscript c). The viscosities are normalized by gas viscosity, i.e. $\psi_{2}^{t^{*}}=\psi_{2}^{t} / \mu$ and $\psi_{2}^{c^{*}}=\psi_{2}^{c} / \mu$. Particles are $200 \mu \mathrm{m}$ diameter and mass loading ratio, $m=3.2$. Other parameters as in Tsuji et al. (1984).

Figure 18. Comparison of radial variations of particulate diffusion coefficient components: a) turbulent (superscript t) and b) collisional (superscript $\mathrm{c}$ ). The coefficients are normalized by the gas diffusion coefficient, i.e. $\kappa_{2}^{t^{*}}=\kappa_{2}^{t} / \kappa$ and $\kappa_{2}^{c^{*}}=\kappa_{2}^{c} / \kappa$, where the diffusion coefficient, $\kappa$, for gas is $0.21 \mathrm{~m}^{2} \mathrm{~s}^{-1}$ (the value for air at atmosphere pressure and $20^{\circ} \mathrm{C}$ ). Particles are 200 $\mu \mathrm{m}$ diameter and mass loading ratio, a) $m=1.0$ and b) $m=3.2$. Other parameters as in Tsuji et al. (1984). 
Figure 19. Simulation results of radial variation of gas normalized r.m.s. fluctuational axial velocity, $v^{\prime} / v_{c}$, for different inter-particle coefficients of restitution: $e=1.0,0.9$ and 0.8 . The mean particle diameter, $d=200 \mu \mathrm{m}$, other parameters as in Tsuji et al. (1984). The solid mass loading ratio, $m=3.2$; the gas axial velocity at the pipe center, $v_{c}=10.8 \mathrm{~ms}^{-1}$.

Figure 20. Simulation results of radial variation of normalized r.m.s. fluctuational axial velocity in the particulate phase, $u^{\prime} / v_{c}$, for different inter-particle coefficients of restitution: $e=1.0,0.9$ and 0.8 . The mean particle diameter, $d=200 \mu \mathrm{m}$, other parameters as in Tsuji et al. (1984). The solid mass loading ratio, $m=3.2$; the gas axial velocity at the pipe centre, $v_{c}=10.8$ $\mathrm{ms}^{-1}$.

Figure 21. Simulation results of radial variation of normalized r.m.s. fluctuational axial velocity in the particulate phase, $u^{\prime} / v_{c}$, for different inter-particle coefficients of restitution: $e=1.0,0.9$ and 0.8 . The mean particle diameter, $d=200 \mu \mathrm{m}$, other parameters as in Tsuji et al. (1984). The solid mass loading ratio, $m=1.0$; the gas axial velocity at the pipe centre, $v_{c}=18.9$ $\mathrm{ms}^{-1}$.

Figure 22. Simulation results of radial variation of normalized r.m.s. fluctuational axial velocity in a) the gas phase, $v^{\prime} / v_{c}$, and b) the particulate phase, $u^{\prime} / v_{c}$, for different specularity coefficients: $\phi=0.012,0.008,0.004$ and 0.002 . The particle diameter, $d=200 \mu \mathrm{m}$, other parameters as in Tsuji et al. (1984). The solid mass loading ratio, $m=0.9$; the gas axial velocity at the pipe center, $v_{c}=12.8 \mathrm{~ms}^{-1}$.

Figure 23. Simulation results of radial variation of normalized r.m.s. fluctuational axial velocity in the particulate phase, $u^{\prime} / v_{c}$, for different specularity coefficients: $\phi=0.012,0.008$, 0.004 and 0.002 . The mean particle diameter, $d=200 \mu \mathrm{m}$, other parameters as in Tsuji et al. 
(1984). The solid mass loading ratio, $m=4.2$; the gas axial velocity at the pipe center, $v_{c}=14.6$ $\mathrm{ms}^{-1}$.

Figure 24. Simulation results of radial variation of normalized r.m.s. fluctuational axial velocity in a) the gas phase, $v^{\prime} / v_{c}$, and b) the particulate phase, $u^{\prime} / v_{c}$, for different turbulent constant, $c_{3}=1.85,1.95$, and 2.05 . The solid mass loading ratio, $m=0.3, \mathrm{Re}=22,000$, and the particle diameter, $d=136 \mu \mathrm{m}$. Other parameters as in Maeda et al. (1980).

Figure 25. Simulation results of radial variation of normalized r.m.s. fluctuational axial velocity in a) the gas phase, $v^{\prime} / v_{c}$, and b) the particulate phase, $u^{\prime} / v_{c}$, for different turbulent constant, $c_{3}=1.85,1.95$, and 2.05 . The mean solid volume fraction, $\varepsilon_{2}=5.8 \times 10^{-4}$, the gas velocity at the pipe center, $v_{c}=5.7 \mathrm{~ms}^{-1}$, and the particle diameter, $d=100 \mu \mathrm{m}$. Other parameters as in Lee and Durst (1984).

Figure 26. Simulation results of radial variation of normalized r.m.s. fluctuational axial velocity in a) the gas phase, $v^{\prime} / v_{c}$, and b) the particulate phase, $u^{\prime} / v_{c}$, comparing the effect of including the solid volume fraction at closed packing. The mean solid volume fraction, $\varepsilon_{2}=5.8 \times 10^{-4}$, the gas velocity at the pipe center, $v_{c}=5.7 \mathrm{~ms}^{-1}$, and the particle diameter, $d=100$ $\mu \mathrm{m}$. Other parameters as in Lee and Durst (1984). 


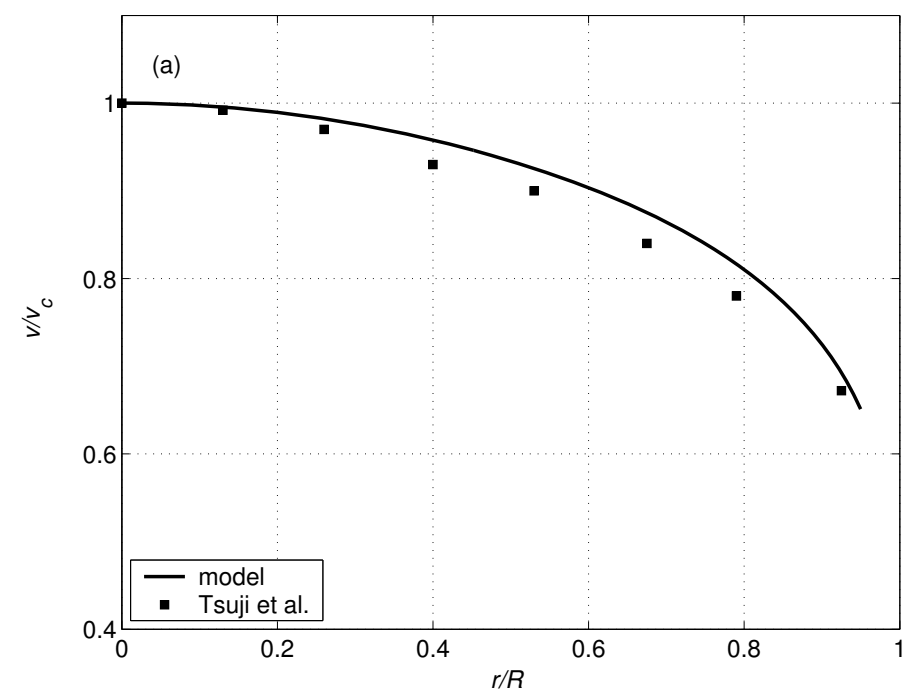




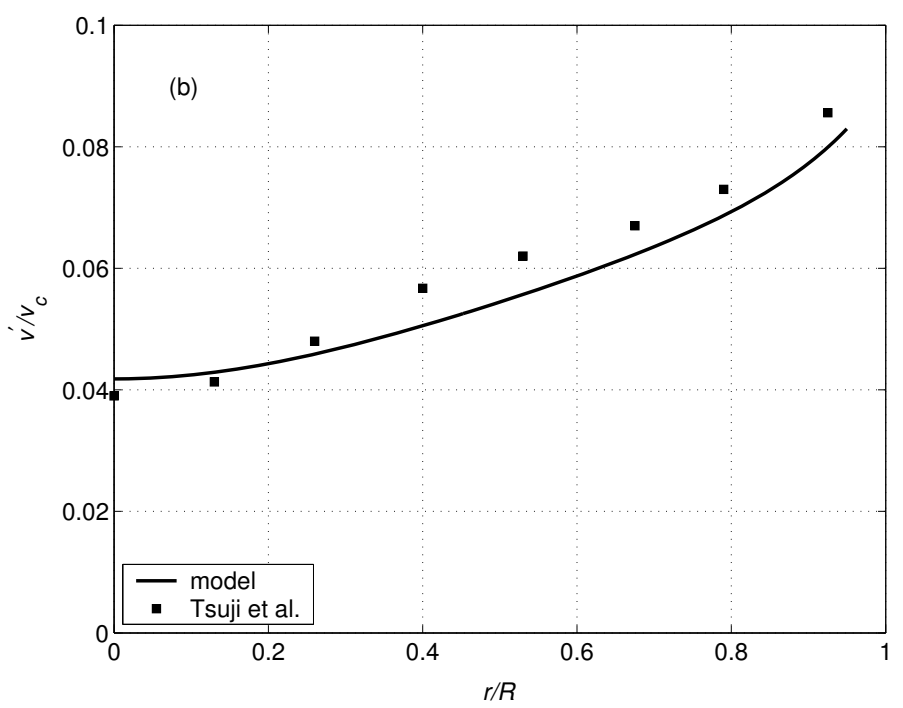

Figure 1. Radial variations of (a) gas normalized axial velocity, $v / v_{c}$, and (b) gas normalized r.m.s. fluctuational axial velocity, $v^{\prime} / v_{c}$, for a pure gas flow. The gas axial velocity at the pipe center, $v_{c}=13.4 \mathrm{~ms}^{-1}$. Other parameters as in Tsuji et al. (1984). 


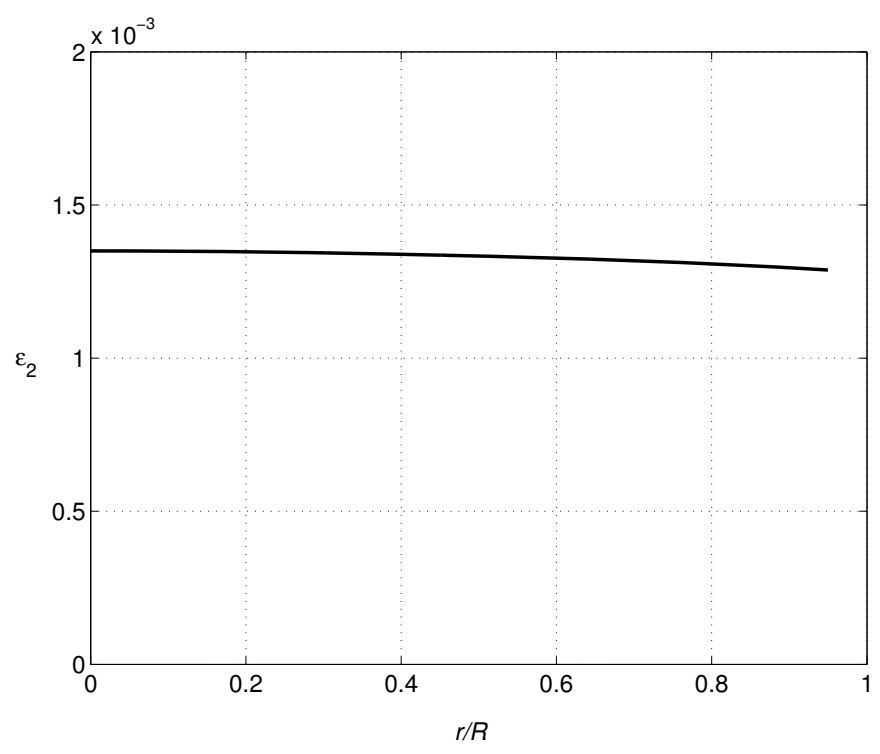

Figure 2. Typical radial variation of particle volume fraction, $\varepsilon_{2}$, in this case for $200 \mu \mathrm{m}$ diameter particles at a mass loading ratio, $m=1.0$. Other parameters as in Tsuji et al. (1984). 


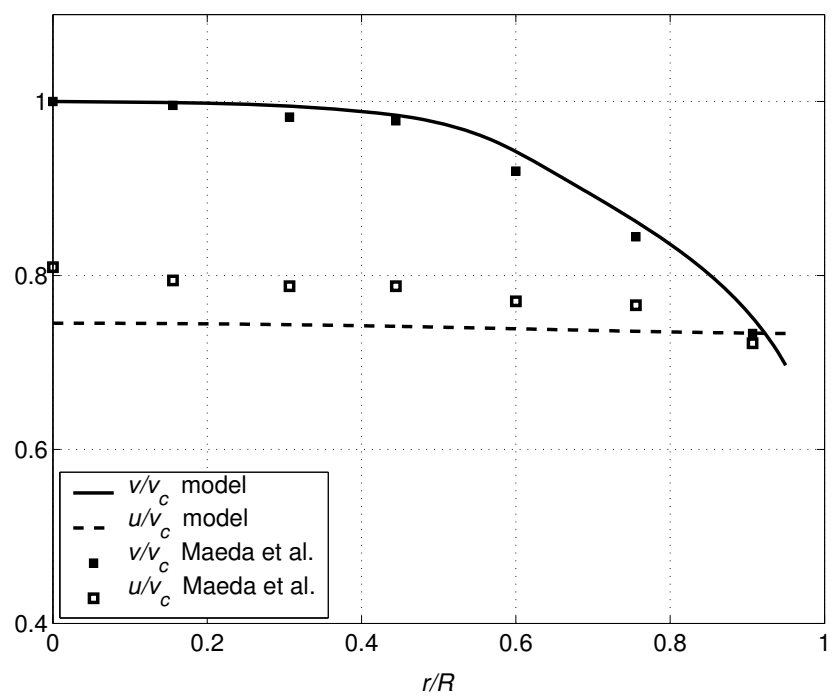

Figure 3. Radial variations of normalized axial velocity of both phases, $v / v_{c}$ and $u / v_{c}$. The mass loading ratio, $m=0.3, \mathrm{Re}=22,000$ and the particles are $136 \mu \mathrm{m}$ diameter. Other parameters as in Maeda et al. (1980). 


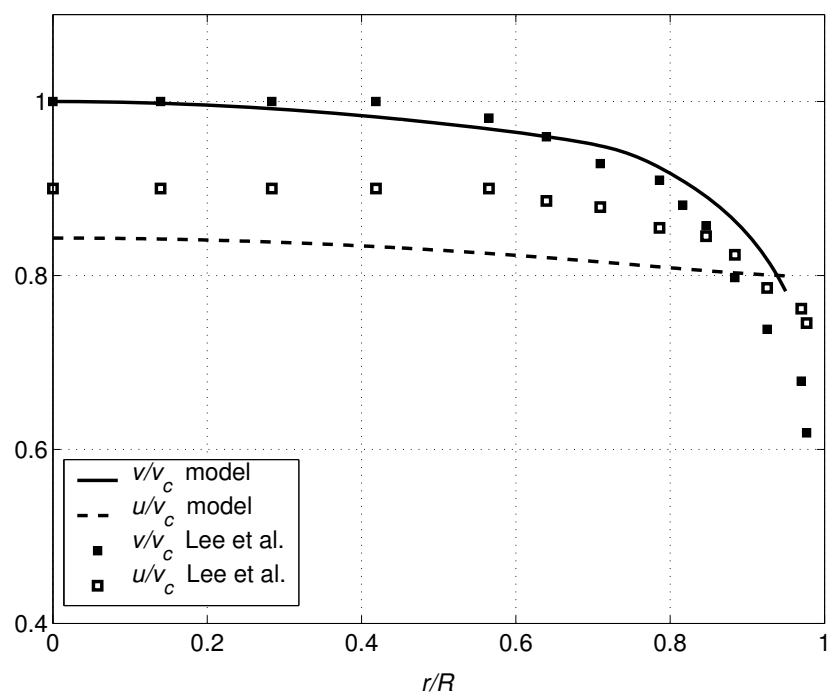

Figure 4. Radial variations of normalized axial velocity of the two phases, $v / v_{c}$ and $u / v_{c}$. The particles are $100 \mu \mathrm{m}$ diameter; the gas axial velocity at the pipe center, $v_{c}=5.7 \mathrm{~ms}^{-1}$; the mean solid volume fraction, $\varepsilon_{2}=5.8 \times 10^{-4}$; and other parameters as in Lee and Durst (1982). 


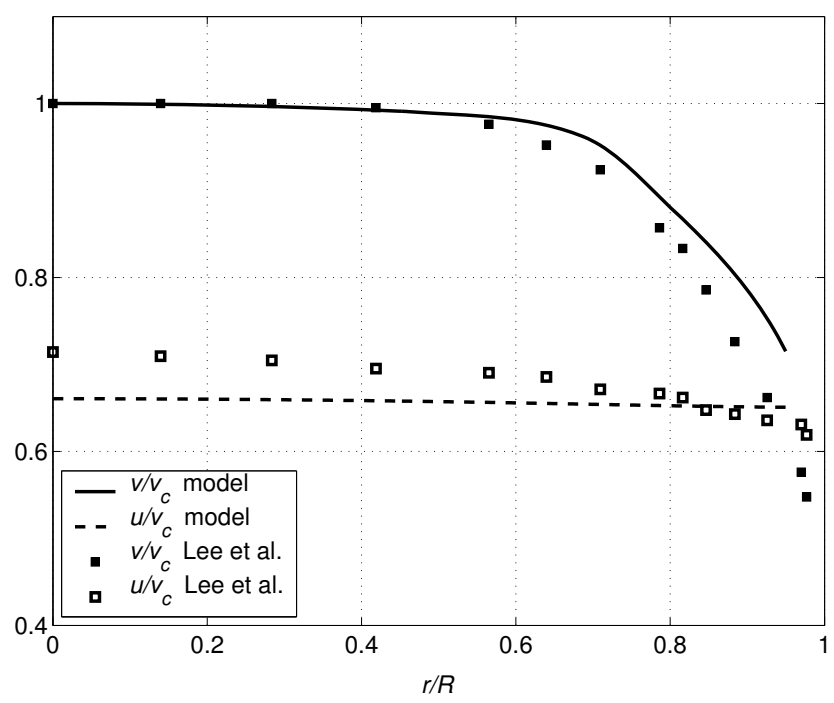

Figure 5. Radial variations of normalized axial velocity of the two phases, $v / v_{c}$ and $u / v_{c}$. The particles are $200 \mu \mathrm{m}$ diameter; the gas axial velocity at the pipe center, $v_{c}=5.84 \mathrm{~ms}^{-1}$; the mean solid volume fraction, $\varepsilon_{2}=6.3 \times 10^{-4}$; and other parameters as in Lee and Durst (1982). 


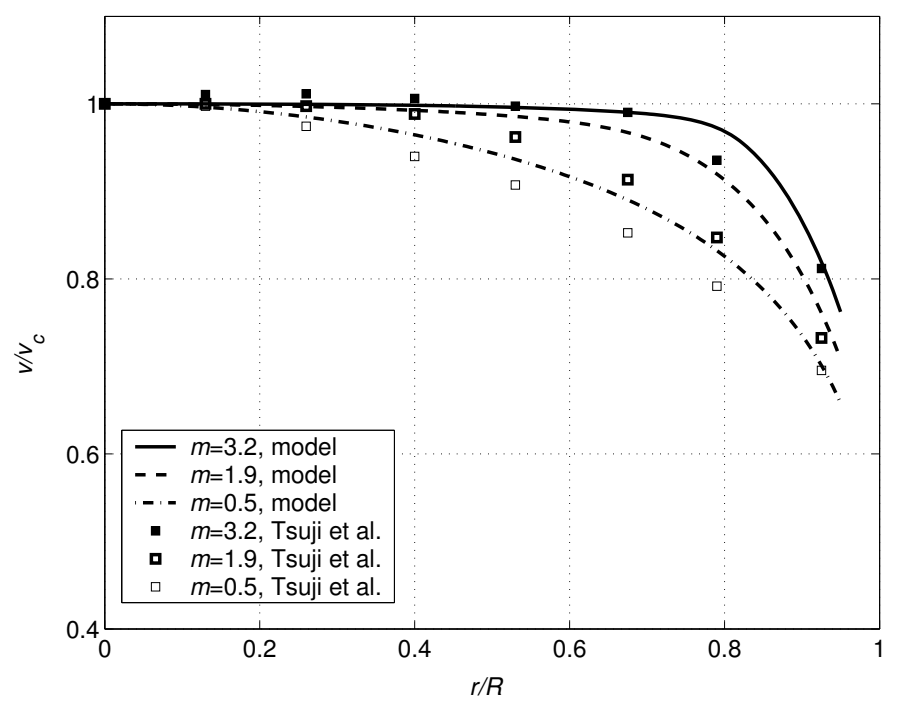

Figure 6. Radial variations of gas normalized axial velocity, $v / v_{c}$. The particles are $200 \mu \mathrm{m}$ diameter. The gas axial velocities at the pipe center, $v_{c}=10.8,11.9$ and $13.1 \mathrm{~ms}^{-1}$ for mass loading ratios, $m=3.2,1.9$ and 0.5 , respectively. Other parameters as in Tsuji et al. (1984). 


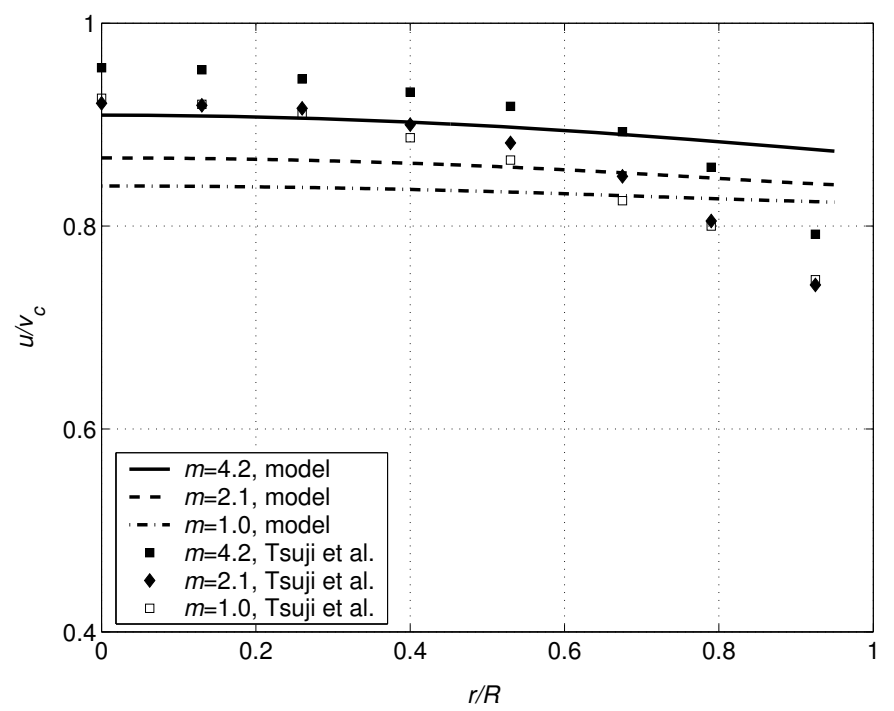

Figure 7. Radial variations of normalized axial velocity of the particulate phase, $u / v_{c}$. The particles are $200 \mu \mathrm{m}$ diameter. The gas axial velocities at the pipe center, $v_{c}=14.6,17.4$ and $18.9 \mathrm{~ms}^{-1}$ for mass loading ratios, $m=4.2,2.1$ and 1.0 , respectively. Other parameters as in Tsuji et al. (1984). 


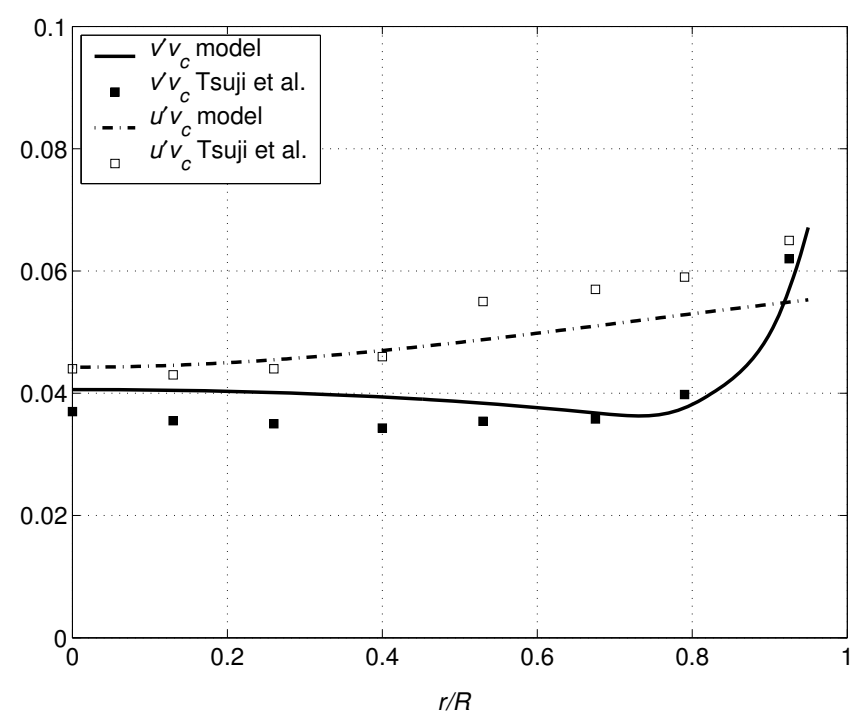

Figure 8. Radial variations of normalized axial r.m.s fluctuational velocity of the two phases, $v^{\prime} / v_{c}$ and $u^{\prime} / v_{c}$. The particles are $200 \mu \mathrm{m}$ diameter. The gas axial velocity at the pipe centre, $v_{c}=10.8 \mathrm{~ms}^{-1}$, and mass loading ratio, $m=3.2$. Other parameters as in Tsuji et al. (1984). 


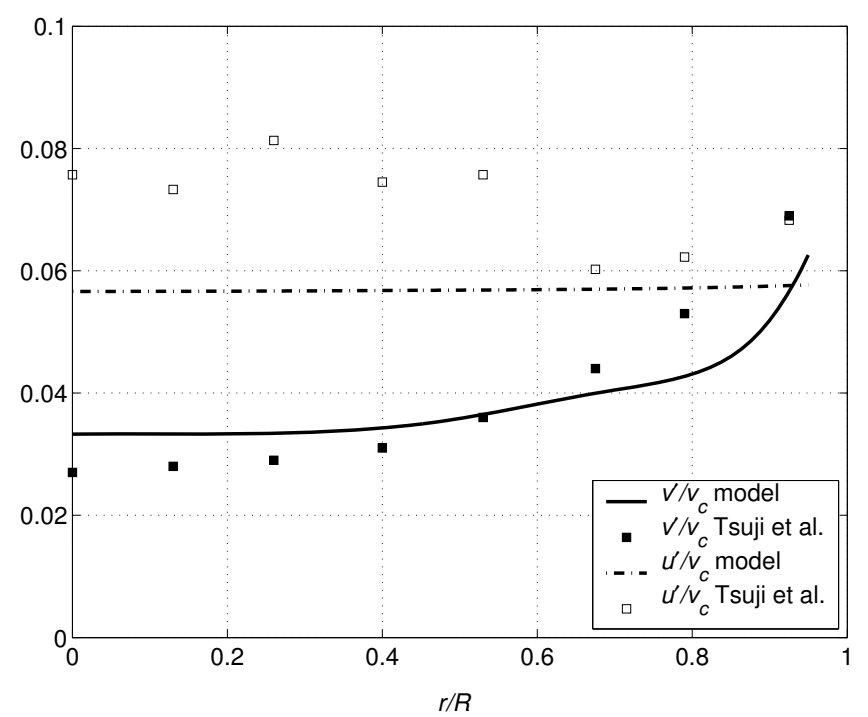

Figure 9. Radial variations of normalized axial r.m.s fluctuational velocity of the two phases, $v^{\prime} v_{c}$ and $u^{\prime} / v_{c}$. The particles are $200 \mu \mathrm{m}$ diameter. The gas axial velocity at the pipe center, $v_{c}=12.8 \mathrm{~ms}^{-1}$, and mass loading ratio, $m=1.3$. Other parameters as in Tsuji et al. (1984). 


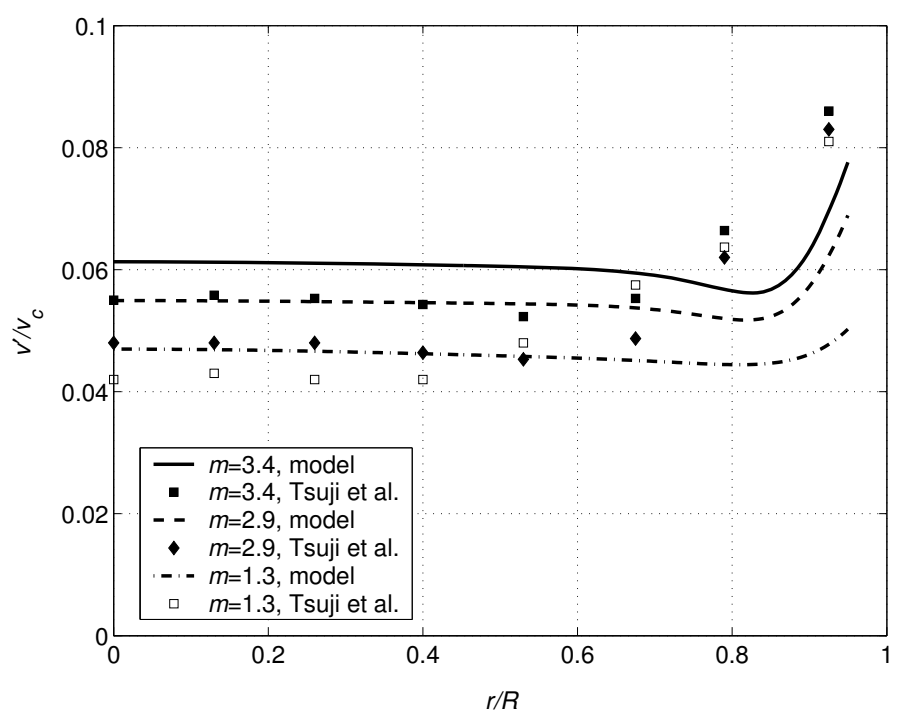

Figure 10. Radial variations of gas normalized axial r.m.s fluctuational velocity, $v^{\prime} / v_{c}$. The particles are $500 \mu \mathrm{m}$ diameter. The gas axial velocities at the pipe center, $v_{c}=10.7,11.4$ and $13.3 \mathrm{~ms}^{-1}$ for mass loading ratios, $m=3.4,2.9$ and 1.3 respectively. Other parameters as in Tsuji et al. (1984). 


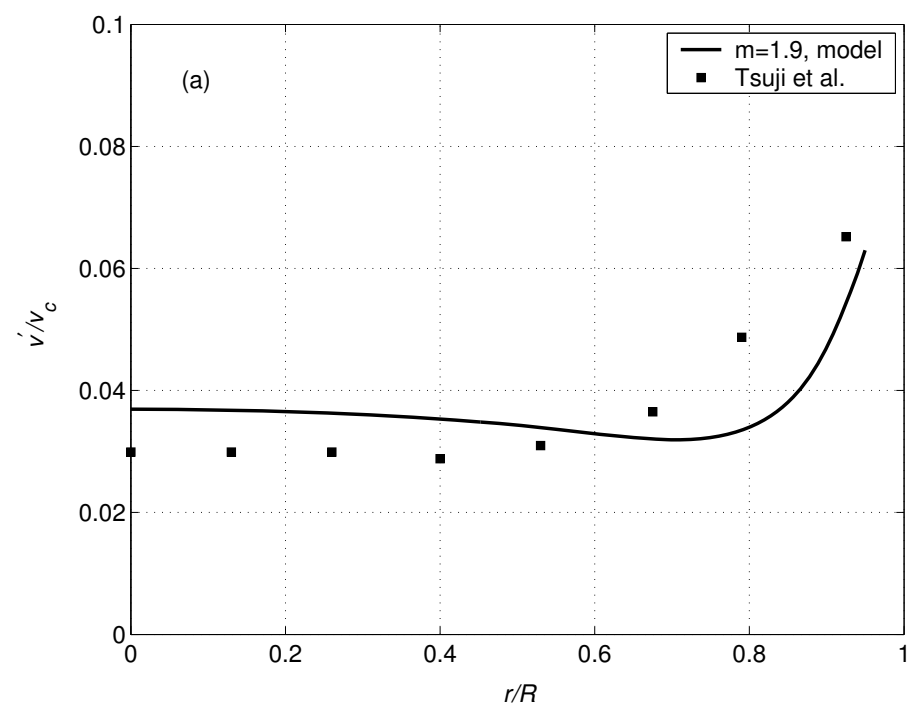




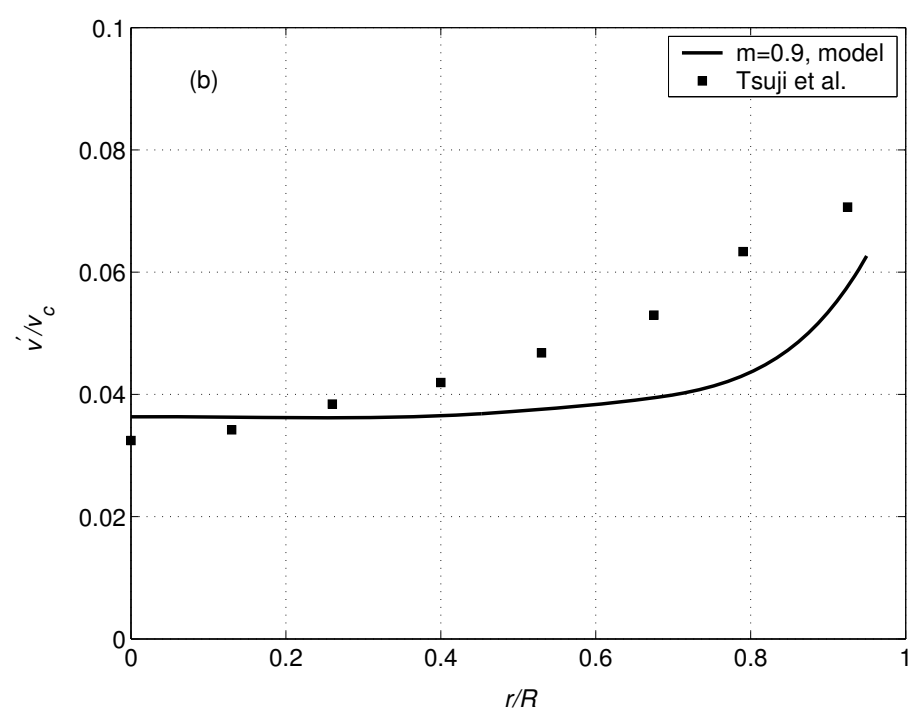

Figure 11. Radial variations of gas normalized axial r.m.s fluctuational velocity, $v^{\prime} / v_{c}$. The particles are $200 \mu \mathrm{m}$ diameter. The gas axial velocities at the pipe center and mass loading ratios are (a) $v_{c}=11.9 \mathrm{~ms}^{-1}, m=1.9$; and (b) $v_{c}=12.8 \mathrm{~ms}^{-1}, m=0.9$. Other parameters as in Tsuji et al. (1984). 


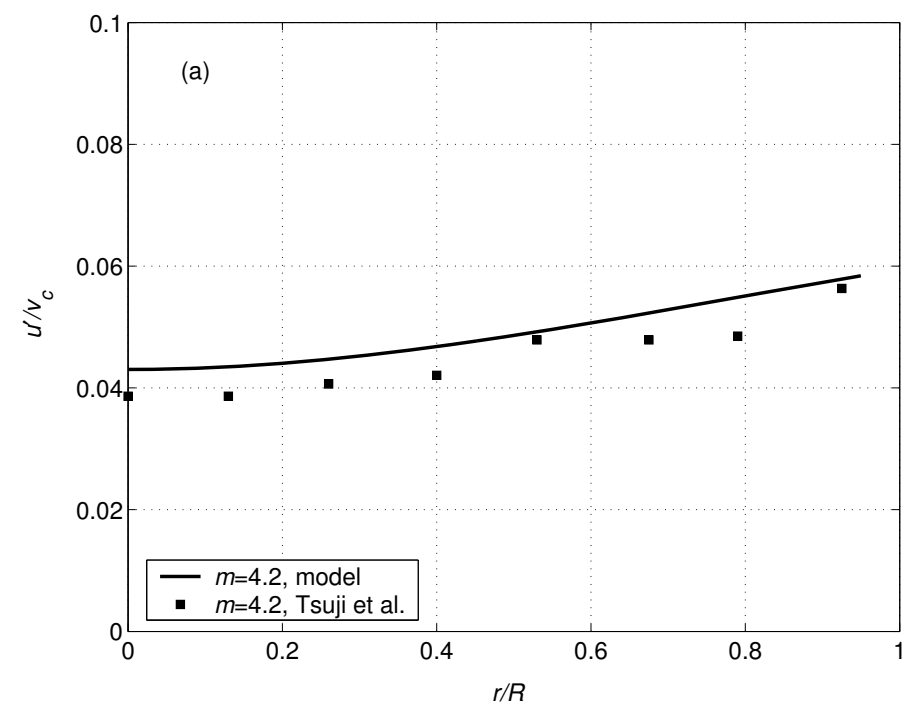




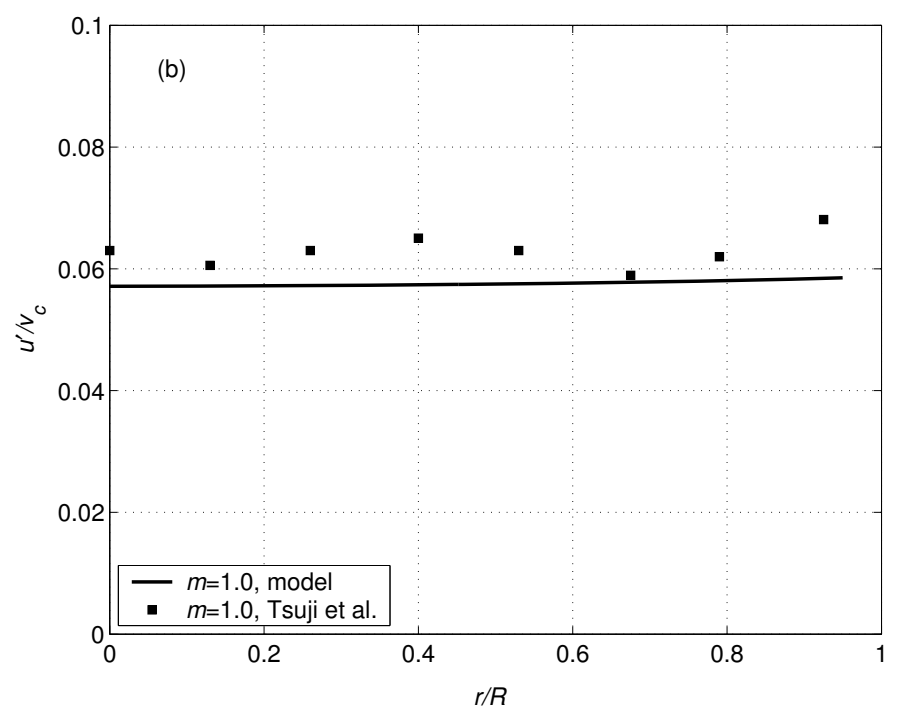

Figure 12. Radial variations of normalized axial r.m.s fluctuational velocity of the particulate phase, $u^{\prime} / v_{c}$. The particles are $200 \mu \mathrm{m}$ diameter. The gas axial velocities at the pipe center and mass loading ratios are (a) $v_{c}=14.6 \mathrm{~ms}^{-1}, m=4.2$; and (b) $v_{c}=18.9 \mathrm{~ms}^{-1}, m=1.0$. Other parameters as in Tsuji et al. (1984). 


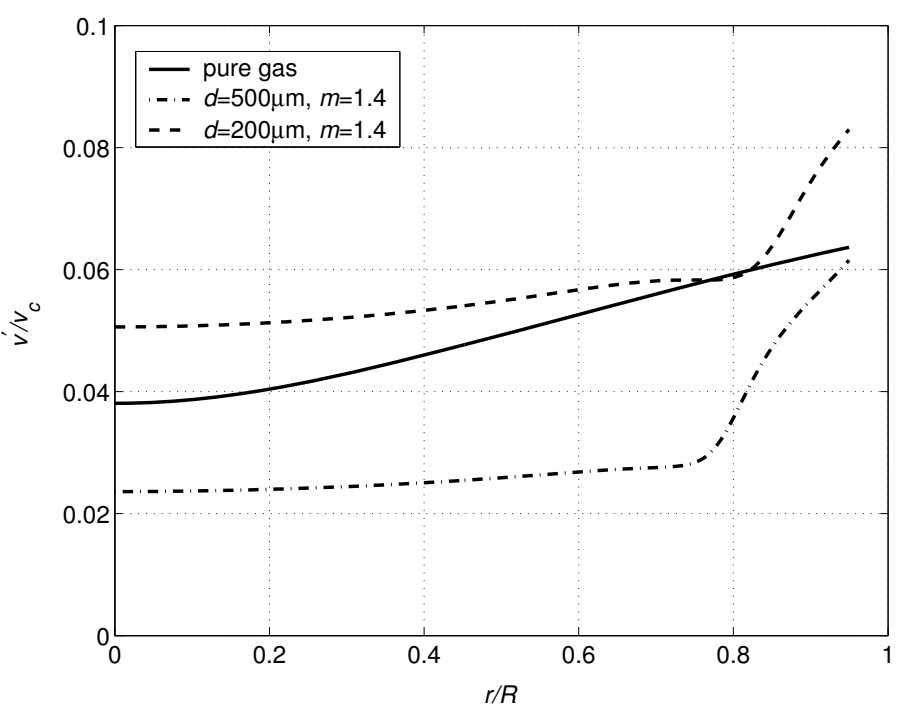

Figure 13. Simulation results for radial variations of gas normalized r.m.s. fluctuational axial velocity, $v^{\prime} / v_{c}$. The gas axial velocity at the pipe center, $v_{c}=5.84 \mathrm{~ms}^{-1}$. For particle-laden flow, the mass loading, $m=1.4$, and the particles are 200 and $500 \mu \mathrm{m}$ diameter. Other parameters as in Lee and Durst (1982). 


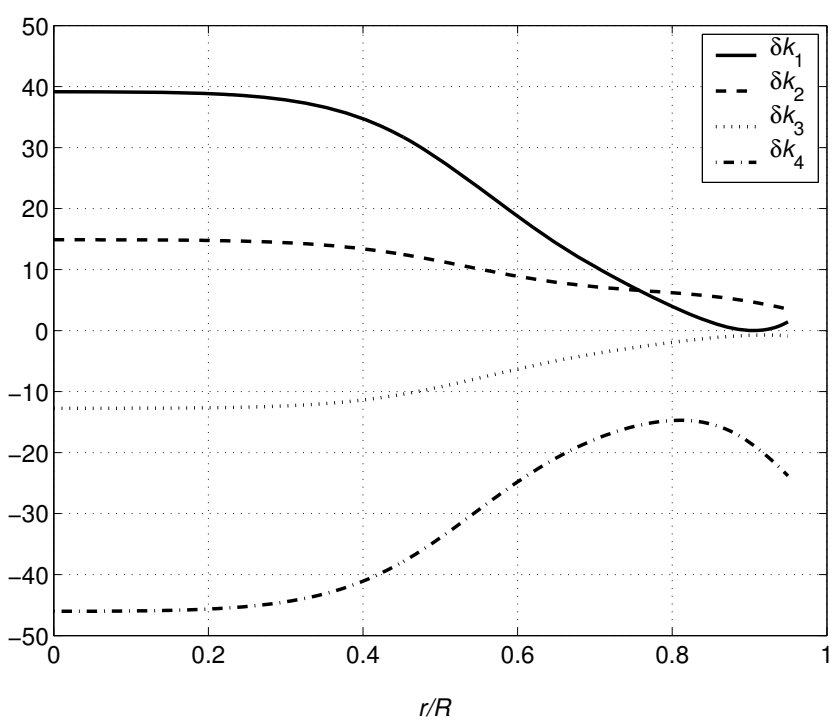

Figure 14. Comparison of radial variation of normalized turbulent energy source and sink terms: $\delta k_{1}=(\boldsymbol{U}-\boldsymbol{V})^{2} / k, \delta k_{2}=3 T / k, \delta k_{3}=-k_{12} / k$ and $\delta k_{4}=-\varepsilon_{1} \rho_{1} \varepsilon / \beta_{0} k$. The particle diameter is $200 \mu \mathrm{m}$ and mass loading ratio, $m=1.0$. Other parameters as in Tsuji et al. (1984). 


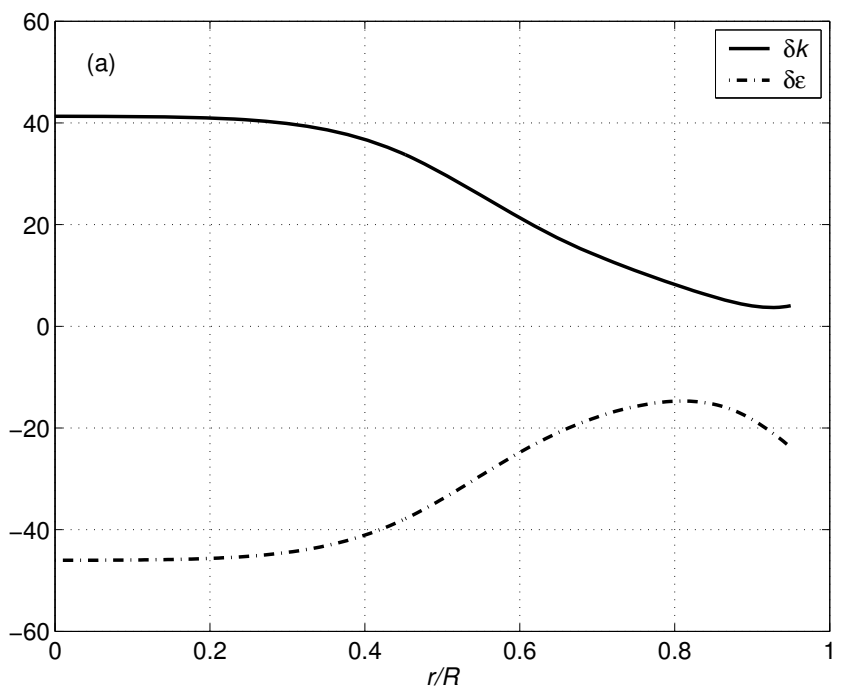




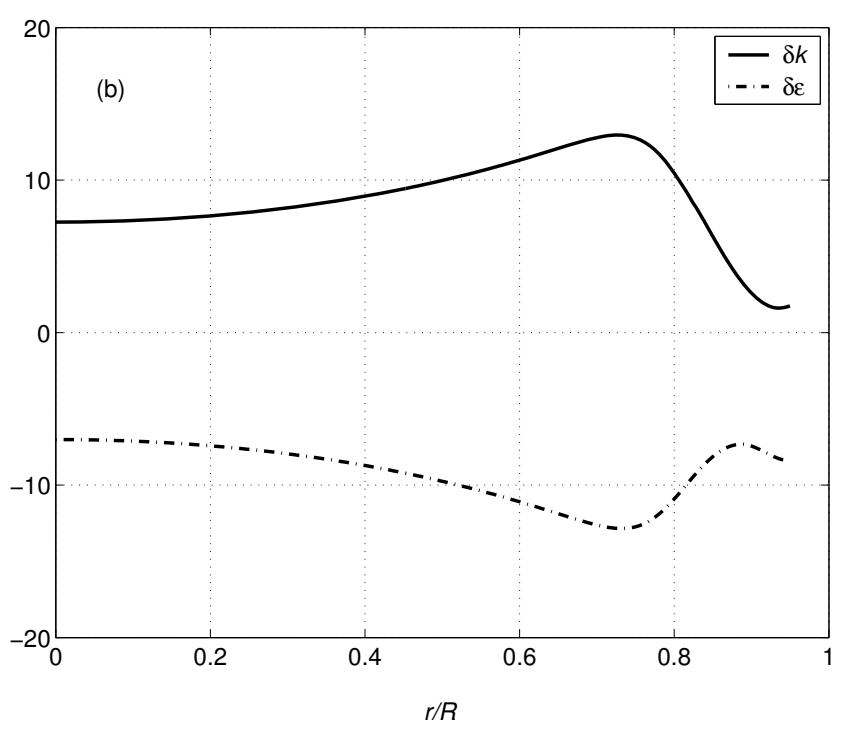

Figure 15 Comparison of normalized turbulent main generation term, due to particles, $\delta k=|\boldsymbol{U}-\boldsymbol{V}|^{2} / k$ and the viscous dissipation term, $\delta \varepsilon=-\varepsilon_{1} \rho_{1} \varepsilon / \beta_{0} k:$ a) $m=1.0$ and b) $m=3.2$, for $200 \mu \mathrm{m}$ particles. Other parameters as in Tsuji et al. (1984). 


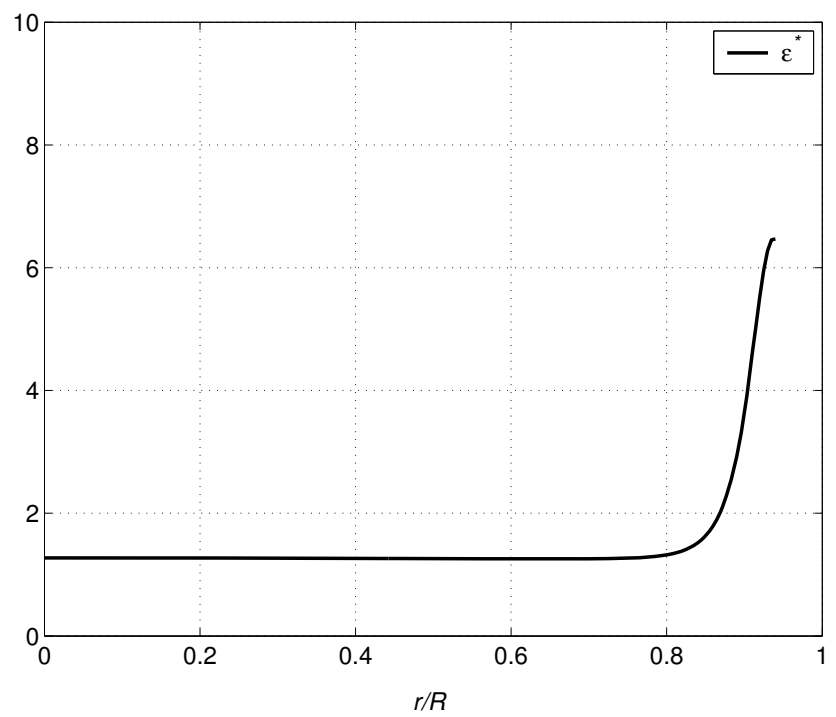

Figure 16. Radial variation of the ratio of the sink, $\delta \varepsilon_{s}=c_{2} \varepsilon_{1} \rho_{1} \varepsilon^{2} / k$, to the source, $\delta \varepsilon_{g}=c_{\varepsilon 3} \beta_{0}\left[|\boldsymbol{U}-\boldsymbol{V}|^{2}+\left(3 T-k_{12}\right)\right] \varepsilon / k$, in equation $(36), \varepsilon^{*}=\delta \varepsilon_{s} / \delta \varepsilon_{g}$. Particle diameter is $200 \mu \mathrm{m}$ and mass loading ratio, $m=3.2$. Other parameters as in Tsuji et al. (1984). 


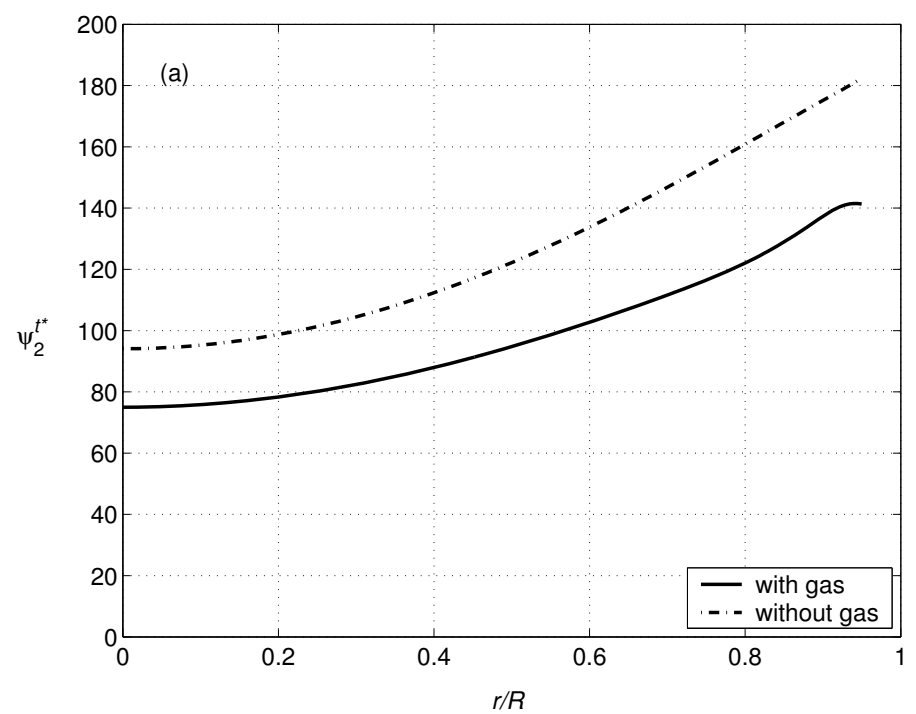




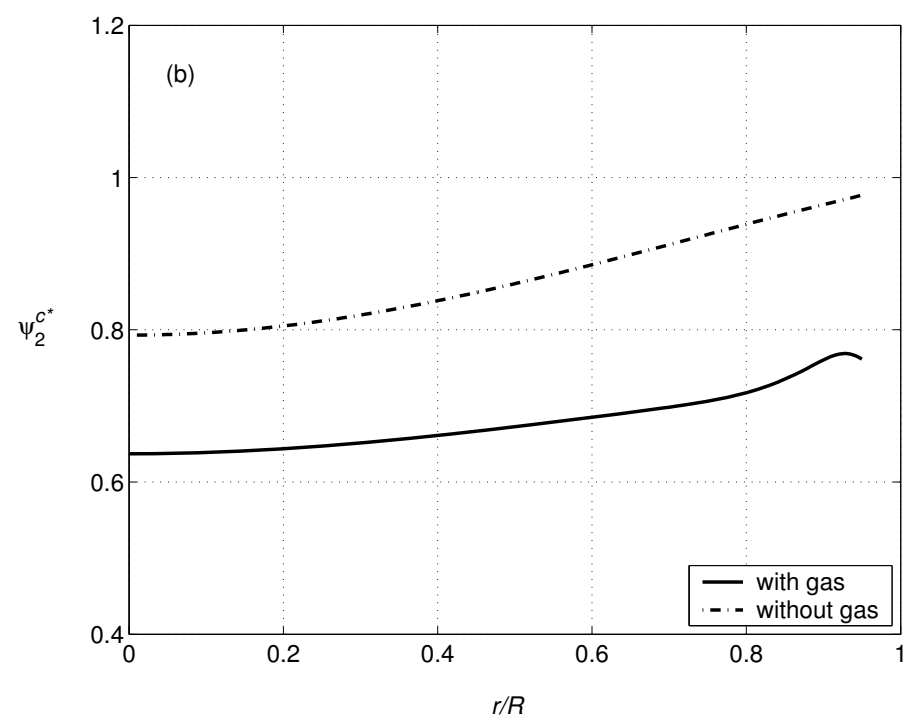

Figure 17. Comparison of radial variations of particulate viscosity components: a) turbulent (superscript t) and b) collisional (superscript c). The viscosities are normalized by gas viscosity, i.e. $\psi_{2}^{t^{*}}=\psi_{2}^{t} / \mu$ and $\psi_{2}^{c^{*}}=\psi_{2}^{c} / \mu$. Particles are $200 \mu \mathrm{m}$ diameter and mass loading ratio, $m=3.2$. Other parameters as in Tsuji et al. (1984). 


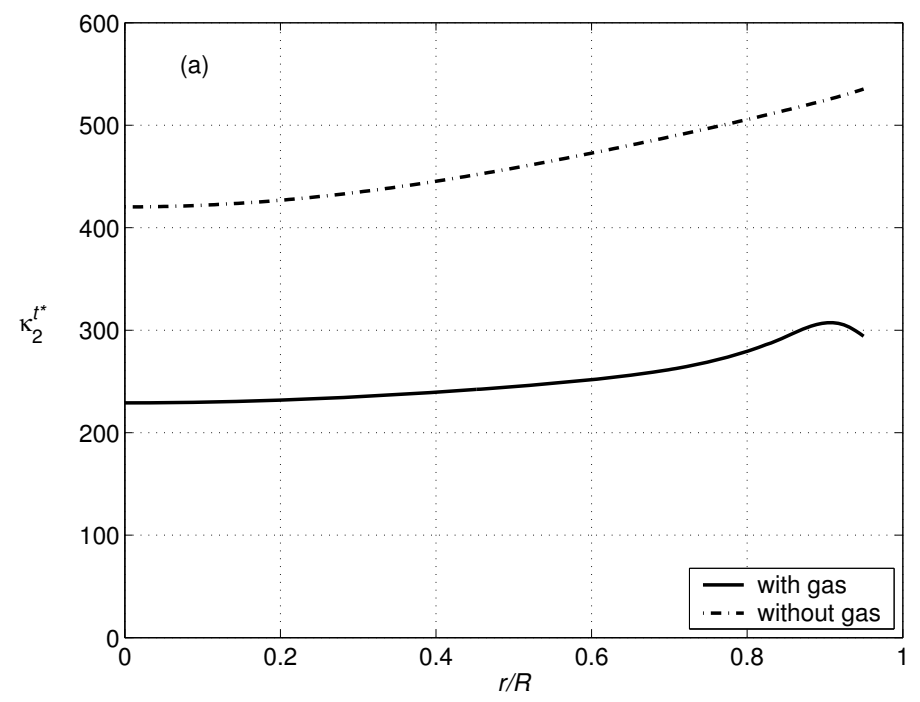




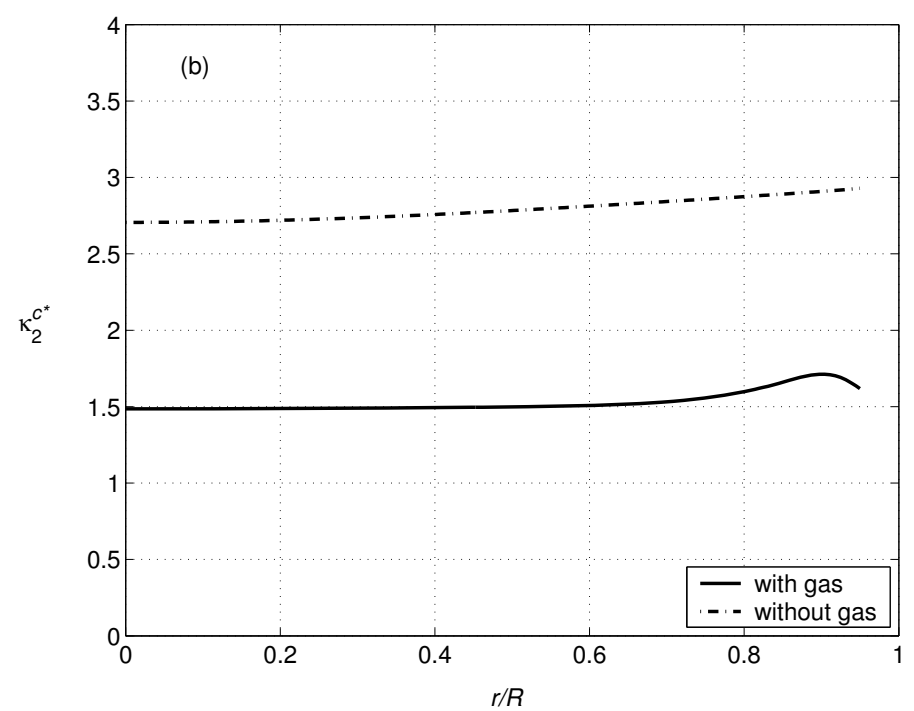

Figure 18. Comparison of radial variations of particulate diffusion coefficient components: a) turbulent (superscript t) and b) collisional (superscript c). The coefficients are normalized by the gas diffusion coefficient, i.e. $\kappa_{2}^{t^{*}}=\kappa_{2}^{t} / \kappa$ and $\kappa_{2}^{c^{*}}=\kappa_{2}^{c} / \kappa$, where the diffusion coefficient, $\kappa$, for gas is $0.21 \mathrm{~m}^{2} \mathrm{~s}^{-1}$ (the value for air at atmosphere pressure and $20^{\circ} \mathrm{C}$ ). Particles are 200 $\mu \mathrm{m}$ diameter and mass loading ratio, a) $m=1.0$ and b) $m=3.2$. Other parameters as in Tsuji et al. (1984). 


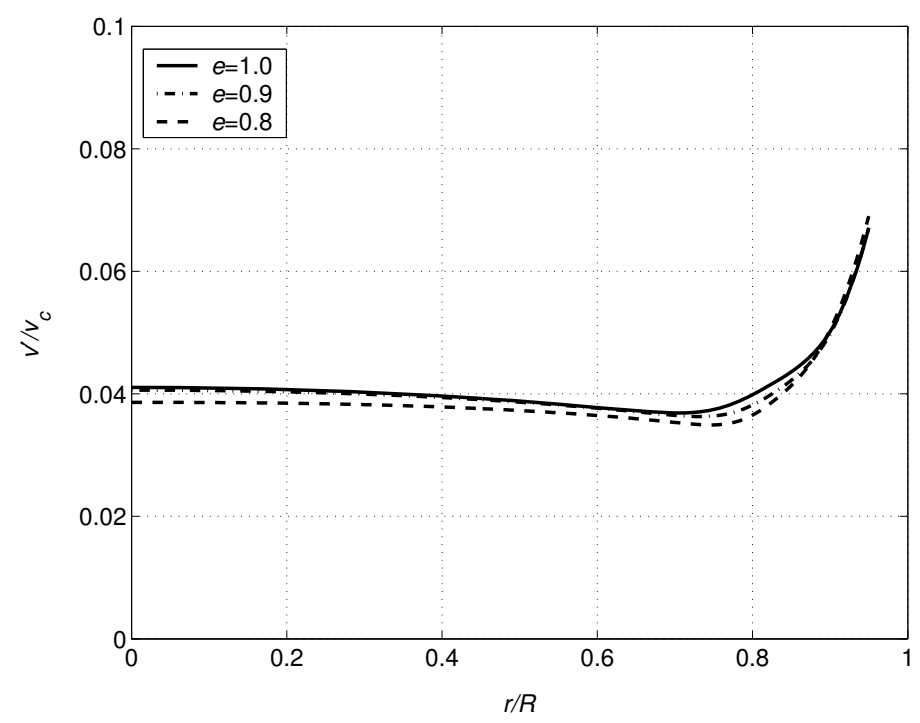

Figure 19. Simulation results of radial variation of gas normalized r.m.s. fluctuational axial velocity, $v^{\prime} / v_{c}$, for different inter-particle coefficients of restitution: $e=1.0,0.9$ and 0.8 . The mean particle diameter, $d=200 \mu \mathrm{m}$, other parameters as in Tsuji et al. (1984). The solid mass loading ratio, $m=3.2$; the gas axial velocity at the pipe center, $v_{c}=10.8 \mathrm{~ms}^{-1}$. 


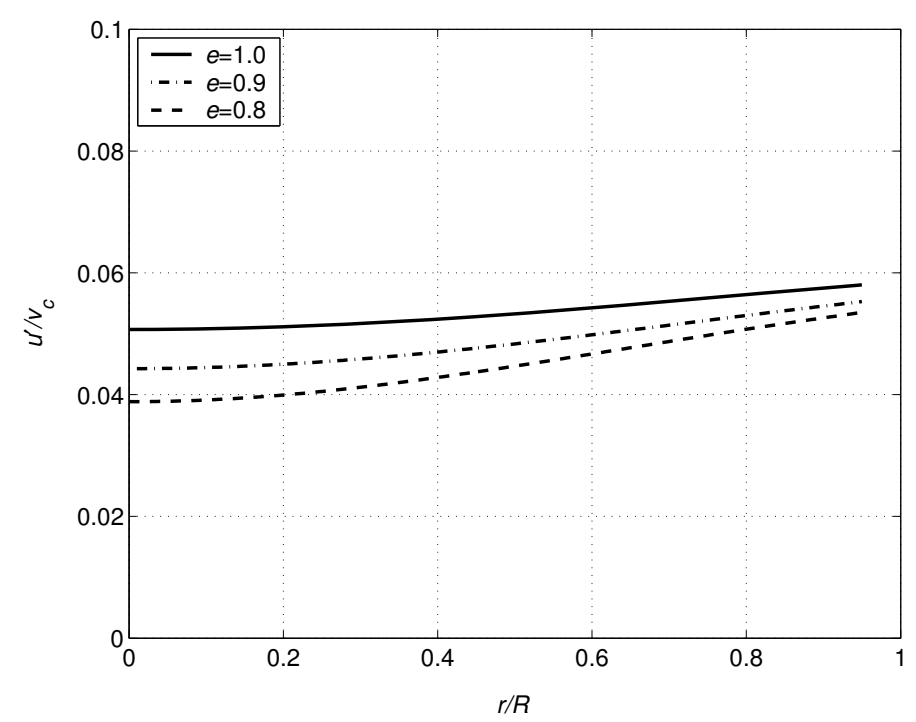

Figure 20. Simulation results of radial variation of normalized r.m.s. fluctuational axial velocity in the particulate phase, $u^{\prime} / v_{c}$, for different inter-particle coefficients of restitution: $e=1.0,0.9$ and 0.8 . The mean particle diameter, $d=200 \mu \mathrm{m}$, other parameters as in Tsuji et al. (1984). The solid mass loading ratio, $m=3.2$; the gas axial velocity at the pipe centre, $v_{c}=10.8$ $\mathrm{ms}^{-1}$. 


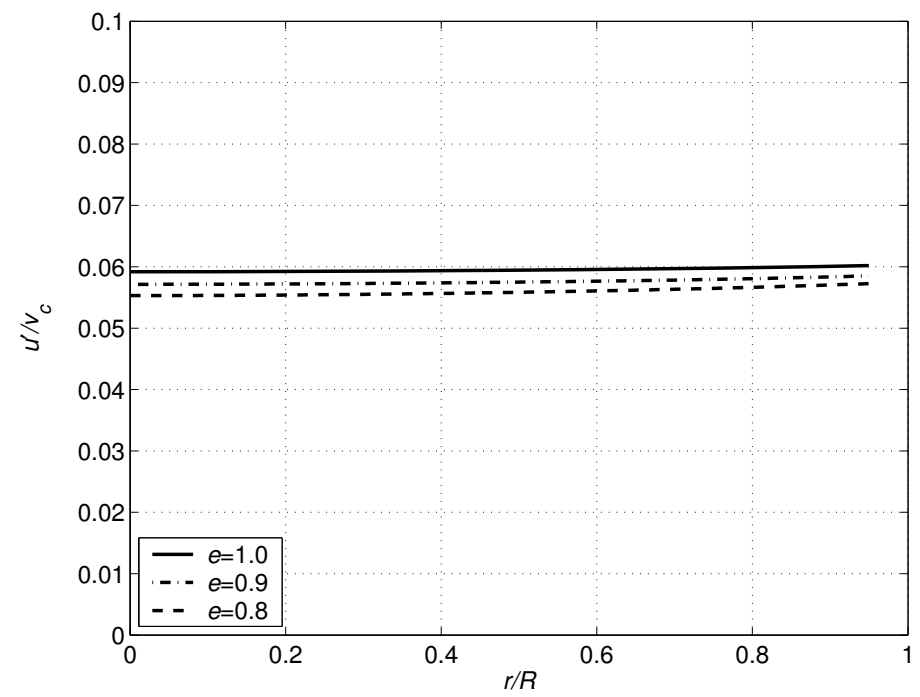

Figure 21. Simulation results of radial variation of normalized r.m.s. fluctuational axial velocity in the particulate phase, $u^{\prime} / v_{c}$, for different inter-particle coefficients of restitution: $e=1.0,0.9$ and 0.8 . The mean particle diameter, $d=200 \mu \mathrm{m}$, other parameters as in Tsuji et al. (1984). The solid mass loading ratio, $m=1.0$; the gas axial velocity at the pipe centre, $v_{c}=18.9$ $\mathrm{ms}^{-1}$. 


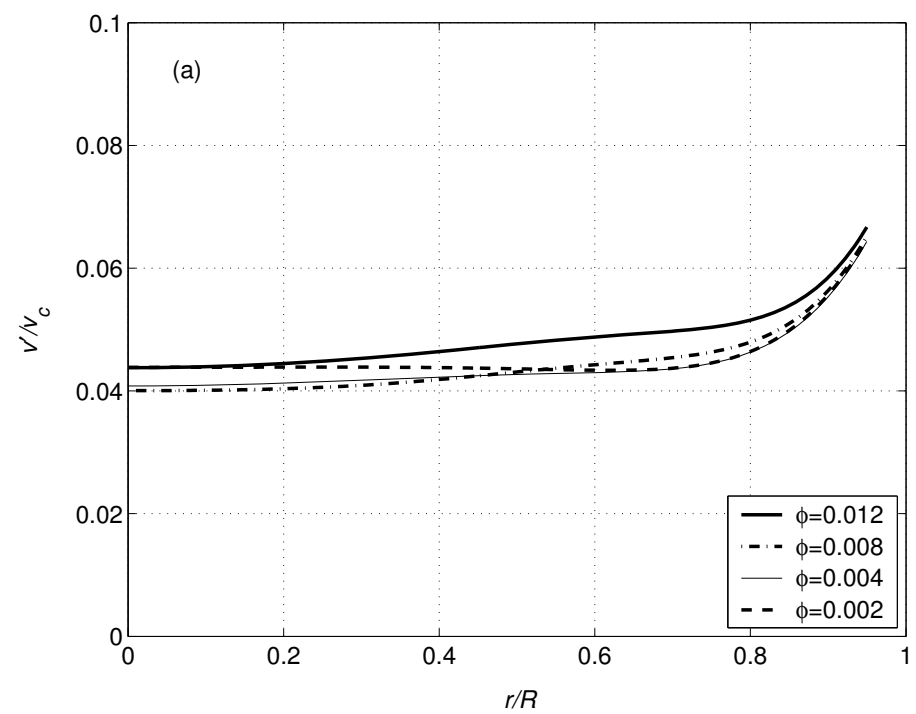




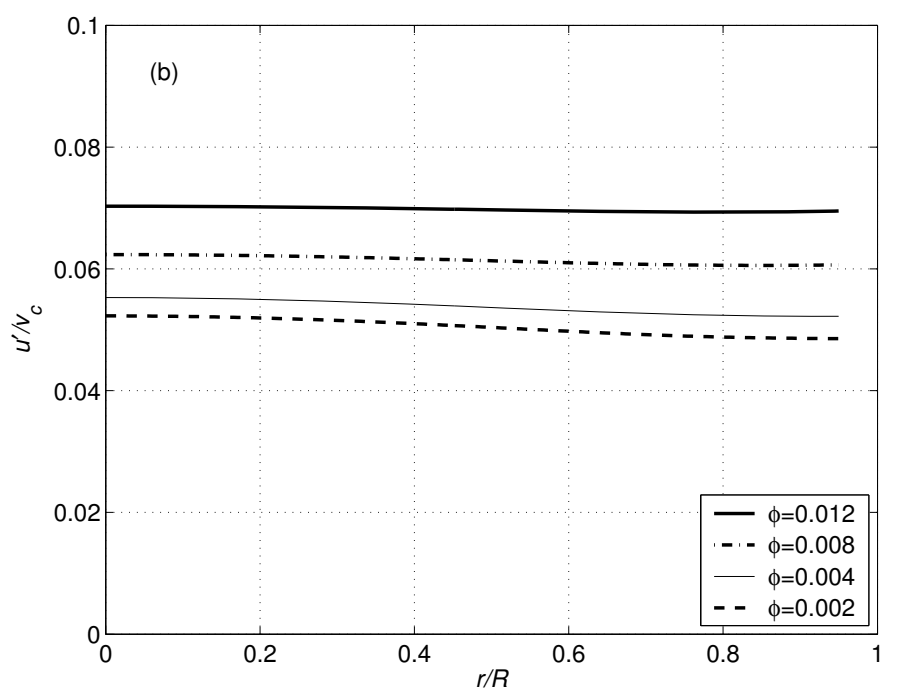

Figure 22. Simulation results of radial variation of normalized r.m.s. fluctuational axial velocity in a) the gas phase, $v^{\prime} / v_{c}$, and b) the particulate phase, $u^{\prime} / v_{c}$, for different specularity coefficients: $\phi=0.012,0.008,0.004$ and 0.002 . The particle diameter, $d=200 \mu \mathrm{m}$, other parameters as in Tsuji et al. (1984). The solid mass loading ratio, $m=0.9$; the gas axial velocity at the pipe center, $v_{c}=12.8 \mathrm{~ms}^{-1}$. 


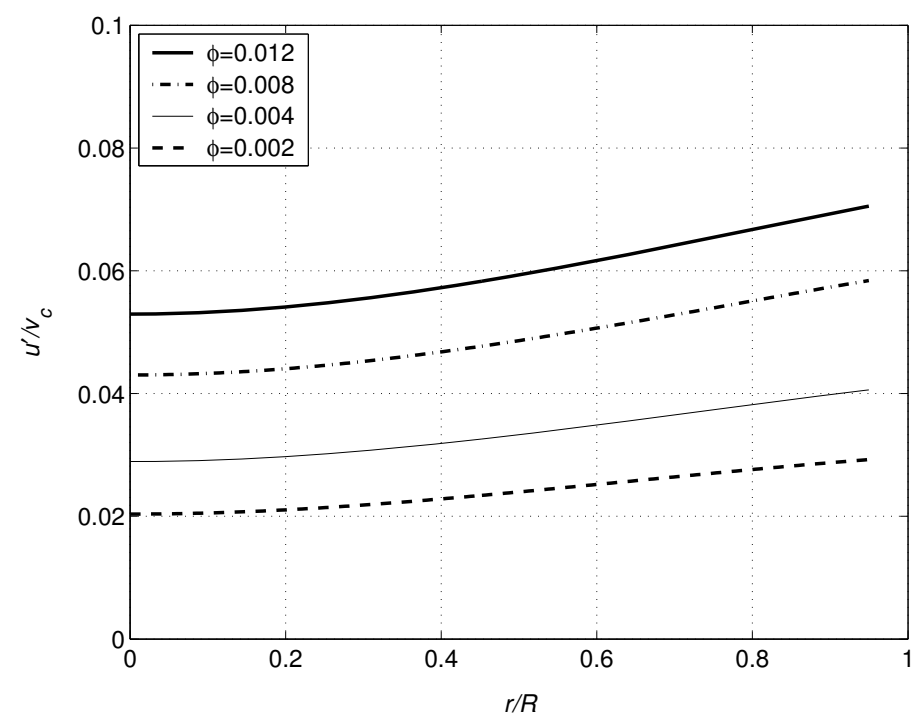

Figure 23. Simulation results of radial variation of normalized r.m.s. fluctuational axial velocity in the particulate phase, $u^{\prime} / v_{c}$, for different specularity coefficients: $\phi=0.012,0.008$, 0.004 and 0.002 . The mean particle diameter, $d=200 \mu \mathrm{m}$, other parameters as in Tsuji et al. (1984). The solid mass loading ratio, $m=4.2$; the gas axial velocity at the pipe center, $v_{c}=14.6$ $\mathrm{ms}^{-1}$. 


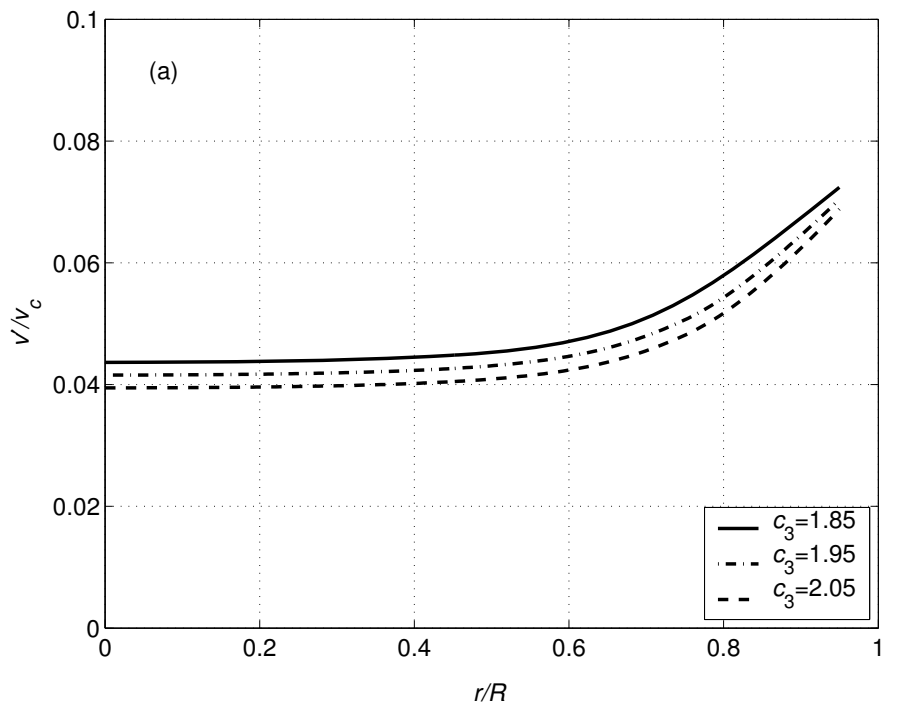




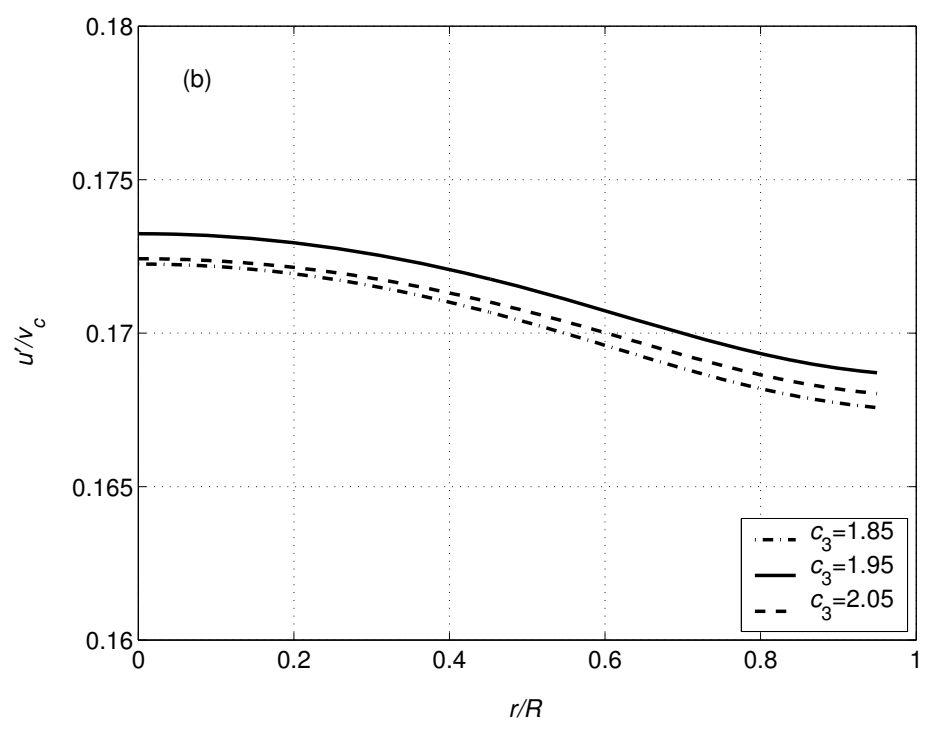

Figure 24. Simulation results of radial variation of normalized r.m.s. fluctuational axial velocity in a) the gas phase, $v^{\prime} / v_{c}$, and b) the particulate phase, $u^{\prime} / v_{c}$, for different turbulent constant, $c_{3}=1.85,1.95$, and 2.05 . The solid mass loading ratio, $m=0.3, \operatorname{Re}=22,000$, and the particle diameter, $d=136 \mu \mathrm{m}$. Other parameters as in Maeda et al. (1980). 


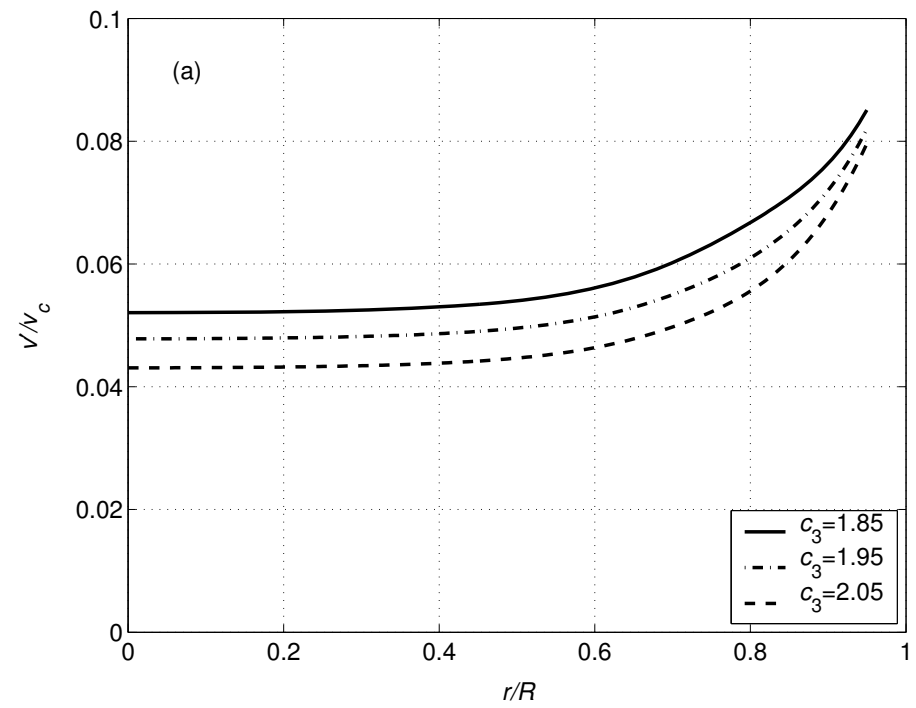




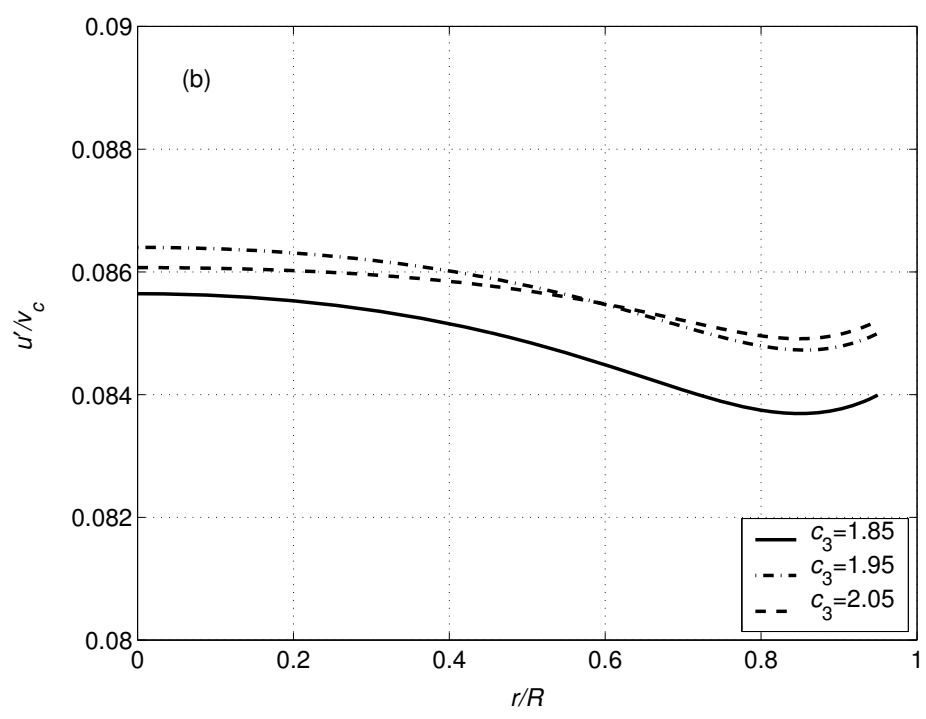

Figure 25. Simulation results of radial variation of normalized r.m.s. fluctuational axial velocity in a) the gas phase, $v^{\prime} / v_{c}$, and b) the particulate phase, $u^{\prime} / v_{c}$, for different turbulent constant, $c_{3}=1.85,1.95$, and 2.05 . The mean solid volume fraction, $\varepsilon_{2}=5.8 \times 10^{-4}$, the gas velocity at the pipe center, $v_{c}=5.7 \mathrm{~ms}^{-1}$, and the particle diameter, $d=100 \mu \mathrm{m}$. Other parameters as in Lee and Durst (1984). 


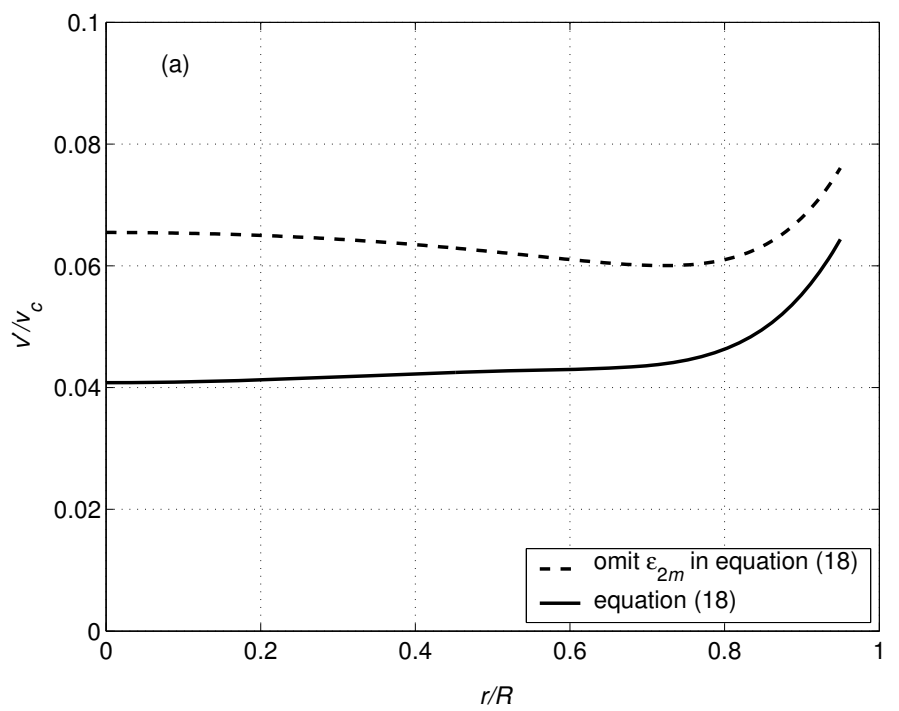




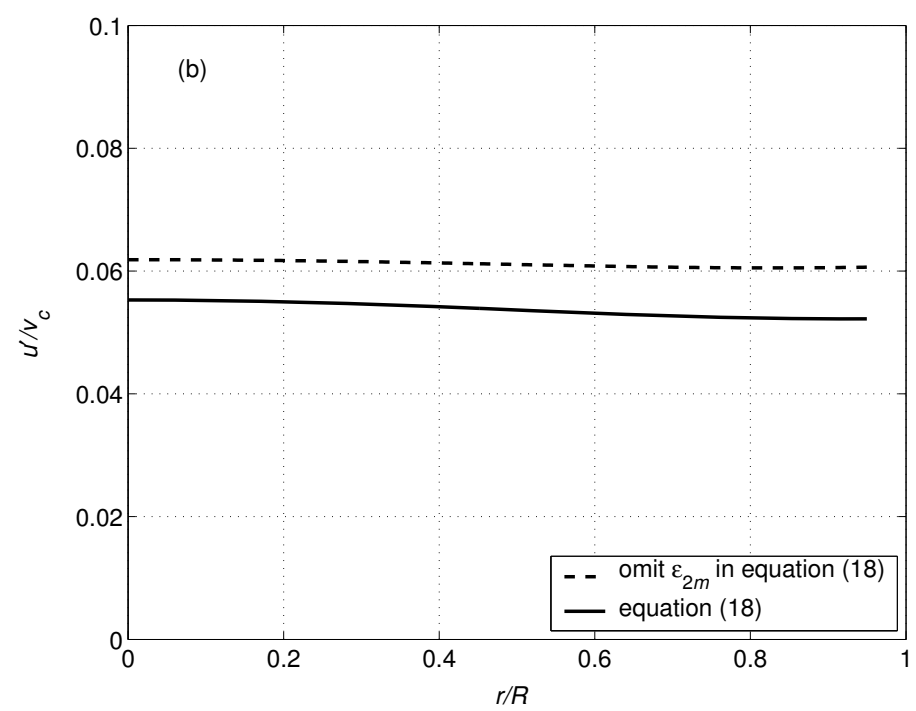

Figure 26. Simulation results of radial variation of normalized r.m.s. fluctuational axial velocity in a) the gas phase, $v^{\prime} / v_{c}$, and b) the particulate phase, $u^{\prime} / v_{c}$, comparing the effect of including the solid volume fraction at closed packing. The mean solid volume fraction, $\varepsilon_{2}=5.8 \times 10^{-4}$, the gas velocity at the pipe center, $v_{c}=5.7 \mathrm{~ms}^{-1}$, and the particle diameter, $d=100$ $\mu \mathrm{m}$. Other parameters as in Lee and Durst (1984). 